\title{
Merging Frameworks for Interaction
}

\author{
Johan van Benthem Jelle Gerbrandy \\ Tomohiro Hoshi Eric Pacuit
}

March 26, 2008

\section{Introduction}

Many logical systems today describe intelligent interacting agents over time. Frameworks include Interpreted Systems (IS, Fagin et al. [8]), Epistemic-Temporal Logic (ETL, Parikh \& Ramanujam [22]), STIT (Belnap et al. [5]), Process Algebra and Game Semantics (Abramsky [1]). This variety is an asset, as different modeling tools can be fine-tuned to specific applications. But it may also be an obstacle, when barriers between paradigms and schools go up.

This paper takes a closer look at one particular interface, between two systems that both address the dynamics of knowledge and information flow in multi-agent systems. One is IS/ETL (IS and ETL are, from a technical point of view, the same up to model transformations, cf. [20]), which uses linear or branching time models with added epistemic structure induced by agents' different capabilities for observing events. These models provide a Grand Stage where histories of some process unfold constrained by a protocol, and a matching epistemic-temporal language describes what happens. The other framework is Dynamic Epistemic Logic (DEL, $[10,4,34])$ that describes interactive processes in terms of epistemic event models which may occur inside modalities of the language. Temporal evolution is then computed from some initial epistemic model through a process of successive 'product updates'. It has long been unclear how to best compare IS/ETL and DEL. Various aspects have been investigated in [10, 30, 32], but in this paper, we study the interface in a more systematic way.

Often, DEL and ETL are presented as alternative ways of adding dynamics to multi-agent epistemic models. In this paper, we rather focus on how merging the two different modeling choices leads to interesting new questions. Our leading interest here will be a view of informational processes as evolving over time.

To see what we mean, consider the simplest version of DEL, viz. the logic of public announcements PAL ([23]) which adds a very specific type of communicative 
action to epistemic models: a public announcement. Formulas of the form $\langle P\rangle \varphi$ are intended to mean "after a public announcement of $P, \varphi$ is true". The $\langle P\rangle$ is interpreted as a restriction of the current model to the states satisfying $P$. Now, in many real interactions between agents, protocol or social convention dictates that some announcements that can happen may not be allowed. For example, in a conversation, it is typically not polite to "blurt everything out at the beginning", as we must speak in small chunks. Other natural protocol rules include 'do not repeat yourself', 'let others speak in turn', 'be honest', and so on. Imposing these rules restricts the legitimate sequences of possible announcements, and this immediately affects the standard validities of PAL. For instance, consider the PAL-validity stating that the effect of two consecutive announcements, expressed in $\langle P\rangle\langle Q\rangle \varphi$, is the same as the effect of one single 'two-in-one' announcement: $(P \wedge$ $\langle P\rangle Q) \varphi$. This equivalence will no longer hold in general protocol-based models, as will be discussed in more detail in Section 4. Other examples of protocols occur in puzzles (the ever-present muddy children are only allowed to make epistemic assertions), while interaction with a database, or some physical measuring device, involves only factual assertions.

In a sense then, this paper is about 'logics of conversation' as governed by protocols. But our results apply much more generally to any sort of informational process, whether linguistically encoded or not. In particular, we want to emphasize another, equally valid interpretation at the outset, which applies to all our notions and results. PAL may also be viewed as a logic of general observation $[28,31]$, without any linguistic communication at all. And then, the protocol setting describes knowledge growth in various learning scenarios, moving closer to formalizing part of the temporal logic of formal learning theory (cf. [17]).

We have two main objectives in this paper. The first is to systematically and rigorously relate the DEL framework with the ETL framework. The key idea is that repeatedly applying product update with sequences of event models creates an ETL model (details are given in the next section). In other words, given an an initial epistemic model and sequences of DEL event models we can generate an ETL model, and thus transform the DEL dynamic modalities into ETL (labeled) temporal modalities. This provides a concrete way of relating DEL and ETL, but it is not the whole story. The precise relationship between DEL and ETL is explored further in Section 3. We prove a new representation theorem characterizing the largest class of ETL models corresponding to DEL protocols in terms of notions of Perfect Recall, No Miracles, and Bisimulation Invariance. These describe the sort of idealized agent presupposed in standard DEL.

Our second objective is to show how ETL and DEL lead to interesting new issues when merged as accounts of intelligent interacting agents. In particular, we focus on new issues of completeness. One contribution is an axiomatization for 
the dynamic logic of public announcements constrained by protocols, which has been an open problem for some years, as it does not fit the usual 'reduction axiom' format of DEL (cf. Section 4.1.2). More generally, Section 6 provides a number of examples that show how DEL suggests an interesting fine-structure inside ETL.

\section{Relating the Two Frameworks}

In this Section, we give the formal details needed to rigorously compare the DEL and ETL frameworks. We start by fixing a finite set of agents $\mathcal{A}$ and a (possibly infinite) set of events $\Sigma$.

Epistemic Temporal Logic. A history is a finite sequence of events from $\Sigma$. We write $\Sigma^{*}$ for the set of histories built from elements of $\Sigma$. For a history $h$, we write $h e$ for the history $h$ followed by the event $e$. Given $h, h^{\prime} \in \Sigma^{*}$, we write $h \preceq h^{\prime}$ if $h$ is a prefix of $h^{\prime}$, and $h \prec_{e} h^{\prime}$ if $h^{\prime}=h e$ for some event $e$.

Definition 2.1 (ETL Frames) Let $\Sigma$ be a set of events. A protocol is a set $\mathrm{H} \subseteq \Sigma^{*}$ closed under non-empty prefixes. An ETL frame is a tuple $\left\langle\Sigma, \mathrm{H},\left\{\sim_{i}\right.\right.$ \}$\left._{i \in \mathcal{A}}\right\rangle$ with $\mathrm{H}$ a protocol, and for each $i \in \mathcal{A}$, a binary relation $\sim_{i}$ on $^{1} \mathrm{H}$. $\triangleleft$

An ETL frame describes how knowledge evolves over time in some informational process. The protocol captures the temporal structure, with $h^{\prime}$ such that $h \prec_{e} h^{\prime}$ representing the point in time after $e$ has happened in $h$. The relations $\sim_{i}$ represent the uncertainty of the agents about how the current history has evolved. Thus, $h \sim_{i} h^{\prime}$ means that from agent $i$ 's point of view, the history $h^{\prime}$ looks the same as the history $h$.

Different modal languages describe these structures (see, for example, [15, 8]), with 'branching' or 'linear' variants. Here we give just the bare necessities (further language extensions are explored in Section 5). Let At be a countable set of atomic propositions. The language $\mathcal{L}_{E T L}$ is generated by the following grammar:

$$
P|\neg \varphi| \varphi \wedge \psi|[i] \varphi|\langle e\rangle \varphi
$$

where $i \in \mathcal{A}, e \in \Sigma$ and $P \in$ At. The usual boolean connectives $(\vee, \rightarrow, \leftrightarrow)$ and the dual modal operators $(\langle i\rangle,[e])$ are defined as usual. The pure epistemic language, denoted $\mathcal{L}_{E L}$, is the fragment of $\mathcal{L}_{E T L}$ with only epistemic modalities. Formulas are interpreted at histories in an ETL model:

Definition 2.2 (ETL Model) An ETL model is a tuple $\left\langle\Sigma, \mathrm{H},\left\{\sim_{i}\right\}_{i \in \mathcal{A}}, V\right\rangle$ with $\left\langle\Sigma, \mathrm{H},\left\{\sim_{i}\right\}_{i \in \mathcal{A}}\right\rangle$ an ETL frame and $V$ a valuation function $\left(V:\right.$ At $\left.\rightarrow 2^{\mathrm{H}}\right) . \triangleleft$

\footnotetext{
${ }^{1}$ Although we will not do so here, typically it is assumed that $\sim_{i}$ is an equivalence relation.
} 
Definition 2.3 (Truth of $\mathcal{L}_{E T L}$ Formulas) Let $\mathcal{H}=\left\langle\Sigma, \mathrm{H},\left\{\sim_{i}\right\}_{i \in \mathcal{A}}, V\right\rangle$ be an ETL model. The truth of a formula $\varphi$ at a history $h \in \mathrm{H}$, denoted $\mathcal{H}, h \models \varphi$, is defined inductively as follows:

1. $\mathcal{H}, h \models p$ iff $h \in V(p)$

2. $\mathcal{H}, h \models \neg \varphi$ iff $\mathcal{H}, h \not \models \varphi$

3. $\mathcal{H}, h \models \varphi \wedge \psi$ iff $\mathcal{H}, h \models \varphi$ and $\mathcal{H}, h \models \psi$

4. $\mathcal{H}, h \models[i] \varphi$ iff for each $h^{\prime} \in \mathrm{H}$, if $h \sim_{i} h^{\prime}$ then $\mathcal{H}, h^{\prime} \models \varphi$

5. $\mathcal{H}, h \models\langle e\rangle \varphi$ iff there exists $h^{\prime} \in \mathrm{H}$ such that $h \prec_{e} h^{\prime}$ and $\mathcal{H}, h^{\prime} \models \varphi \quad \triangleleft$

It is often natural to extend the language $\mathcal{L}_{E T L}$ with group knowledge operators (e.g., common or distributed knowledge) and more expressive temporal operators (e.g., arbitrary future or past modalities). This may lead to high complexity of the validity problem (cf. [14, 32] and Section 5).

Dynamic Epistemic Logic. An alternative account of interactive dynamics was elaborated by $[10,4,28,33]$ and others. From an initial epistemic model, temporal structure evolves as explicitly triggered by informative events.

Definition 2.4 (Epistemic Model) Let $\mathcal{A}$ be a finite set of agents and At a set of atomic propositions. An epistemic model is a tuple $\left\langle W,\left\{R_{i}\right\}_{i \in \mathcal{A}}, V\right\rangle$ where $W$ is a non-empty set, for each $i \in \mathcal{A}, R_{i}$ is a relation ${ }^{2}$ on $W\left(R_{i} \subseteq W \times W\right)$ and $V$ a valuation function $\left(V:\right.$ At $\left.\rightarrow 2^{W}\right)$. We call the set $W$ the domain of $\mathcal{M}$, denoted by $D(\mathcal{M})$. A pair $\mathcal{M}, w$ where $\mathcal{M}$ is an epistemic model and $w \in D(\mathcal{M})$ is called a pointed epistemic model.

We can interpret the epistemic language, $\mathcal{L}_{E L}$, defined above at states in an epistemic model. Truth is defined as usual: see [6] for details. We only recall the definition of the knowledge operators:

$$
\mathcal{M}, w \models[i] \varphi \text { iff for each } w \in W \text {, if } w R_{i} w^{\prime} \text { then } \mathcal{M}, w^{\prime}=\varphi
$$

Whereas an ETL frame describes the agents' information at all moments, event models are used to build new epistemic models as needed.

Definition 2.5 (Event Model, Product Update) An event model $\mathcal{E}$ is a tuple $\left\langle S,\{\longrightarrow\}_{i \in \mathcal{A}}\right.$, pre $\rangle$, where $S$ is a nonempty set, for each $i \in \mathcal{A}, \longrightarrow{ }_{i} \subseteq S \times S$

\footnotetext{
${ }^{2}$ Again, the $R_{i}$ are often taken to be equivalence relations on $W$ - but we do not commit.
} 
and pre $: S \rightarrow \mathcal{L}_{E L}$ is the pre-condition function. The set $S$ is called the domain of $\mathcal{E}$, denoted $D(\mathcal{E})$.

The product update $\mathcal{M} \otimes \mathcal{E}$ of an epistemic model $\mathcal{M}=\left\langle W,\left\{R_{i}\right\}_{i \in \mathcal{A}}, V\right\rangle$ and event model $\mathcal{E}=\left\langle S,\left\{\longrightarrow_{i}\right\}_{i \in \mathcal{A}}\right.$, pre $\rangle$ is the epistemic model $\left\langle W^{\prime}, R_{i}^{\prime}, V^{\prime}\right\rangle$ with

1. $W^{\prime}=\{(w, e) \mid w \in W, e \in S$ and $\mathcal{M}, w \models \operatorname{pre}(e)\}$,

2. $(w, e) R_{i}\left(w^{\prime}, e^{\prime}\right)$ iff $w R_{i} w^{\prime}$ in $\mathcal{M}$ and $e \longrightarrow_{i} e^{\prime}$ in $\mathcal{E}$, and

3. $V^{\prime}((s, e))=V(s)$.

The language $\mathcal{L}_{D E L}$ extends $\mathcal{L}_{E L}$ with operators $\langle\mathcal{E}, e\rangle$ for each pair of event models $\mathcal{E}$ and event $e$ in the domain of $\mathcal{E}$. Truth for $\mathcal{L}_{D E L}$ is defined as usual. We only define the typical DEL modalities: $\mathcal{M}, w \models\langle\mathcal{E}, e\rangle \varphi$ iff $\mathcal{M}, w \models \operatorname{pre}(e)$ and $\mathcal{M} \otimes$ $\mathcal{E},(w, e) \models \varphi$ (see [3] for more details, and [33] for extended versions of product update allowing factual change).

Remark 2.6 (Size of the Event Models) Although Definition 2.5 does not assume that event models are finite, it is often convenient to make such an assumption. The main reason is that the usual reduction axiom for the DEL modality $[\mathcal{E}, e](c f .[4])$ contains a conjunction over all elements of $\mathcal{E}$ reachable from e. Now if this set is infinite, then the reduction axiom will not be a formula of $\mathcal{L}_{D E L}$ since it contains an infinite conjunction. We return to this issue in Section 3.

Example: Public Announcement Logic $([23,11])$. The public announcement of a formula $\varphi \in \mathcal{L}_{E L}$ is the event model $\mathcal{E}_{\varphi}=\left\langle\{e\},\left\{\longrightarrow_{i}\right\}_{i \in \mathcal{A}}\right.$, pre $\rangle$ where for each $i \in \mathcal{A}, e \longrightarrow_{i} e$ and $\operatorname{pre}(e)=\varphi$. The product update of an epistemic model $\mathcal{M}$ with a public announcement model $\mathcal{E}_{\varphi}$ is the submodel of $\mathcal{M}$ containing all the states that satisfy $\varphi$. In this case, the DEL modality $\left\langle\mathcal{E}_{\varphi}, e\right\rangle$ will be denoted $\langle\varphi\rangle$. Henceforth, $\mathcal{L}_{P A L}$ will denote this language.

From DEL Protocols to ETL Models Our key observation is that by repeatedly updating an epistemic model with event models, the machinery of DEL in effect creates ETL models. However, note that an ETL model contains not only a description of how the agents' information changes over time, but also "protocol information" describing when each event can be performed. Thus, in rigorously comparing DEL with ETL models, the protocol information must be made explicit, constraining how the relevant conversation, observational set-up, or learning scenario can evolve.

To make this precise, let $\mathbb{E}$ be the class of all pointed event models, i.e., $\mathbb{E}=\{(\mathcal{E}, e) \mid \mathcal{E}$ an event model and $e \in D(\mathcal{E})\}$. A DEL protocol is a set $\mathrm{P} \subseteq \mathbb{E}^{*}$ 
closed under the initial segment relations (cf. Definition 2.1) ${ }^{3}$. Given a DEL protocol $\mathbf{P}$, let $\sigma$ denote an element of $\mathbf{P}$ (so, $\sigma$ is a sequence of pointed event models). We write $\sigma_{n}$ for the initial segment of $\sigma$ of length $n(n \leq \operatorname{len}(\sigma))$ and write $\sigma_{(n)}$ for the $n$th component of $\sigma$. For example, if $\sigma=\left(\mathcal{E}_{1}, e_{1}\right)\left(\mathcal{E}_{2}, e_{2}\right)\left(\mathcal{E}_{3}, e_{3}\right) \cdots\left(\mathcal{E}_{n}, e_{n}\right)$, then $\sigma_{3}=\left(\mathcal{E}_{1}, e_{1}\right)\left(\mathcal{E}_{2}, e_{2}\right)\left(\mathcal{E}_{3}, e_{3}\right)$ and $\sigma_{(3)}=\left(\mathcal{E}_{3}, e_{3}\right)$. Given a sequence $\sigma \in \mathbb{E}^{*}$, we abuse notation and write $\operatorname{pre}\left(\sigma_{(n)}\right)$ for pre $\left(e_{n}\right)$ where $\sigma_{(n)}=\left(\mathcal{E}_{n}, e_{n}\right)$. Furthermore, we write $\sigma_{(n)} \longrightarrow_{i} \sigma_{(n)}^{\prime}$ provided $\sigma_{(n)}=(\mathcal{E}, e)$ and $\sigma_{(n)}^{\prime}=\left(\mathcal{E}, e^{\prime}\right)$ and $e \longrightarrow_{i} e^{\prime}$ in $\mathcal{E}$. Finally, let $\operatorname{Ptcl}(\mathbb{E})$ be the class of all DEL protocols, i.e., $\operatorname{Ptcl}(\mathbb{E})=\left\{\mathrm{P} \mid \mathrm{P} \subseteq \mathbb{E}^{*}\right.$ is closed under initial segments $\}$.

The main idea is that starting from an initial (pointed) epistemic model we construct an ETL model by repeatedly applying product update. Our most general construction will vary the DEL protocol from state-to-state:

Definition 2.7 (State-Dependent DEL Protocol) Let $\mathcal{M}$ be an arbitrary epistemic model. A state-dependent DEL protocol on $\mathcal{M}$ is any function $p$ : $D(\mathcal{M}) \rightarrow \operatorname{Ptcl}(\mathbb{E})$.

This is a significant generalization of the usual ETL setting where the protocol is assumed to be common knowledge (cf. $[8,22]$ ). If a state-dependent protocol $p$ is a constant function (i.e., for all $w \in D(\mathcal{M}), p(w)=\mathrm{P}$ ), we say $p$ is a uniform DEL protocol. To ease exposition, we will denote a uniform DEL protocol by the unique DEL protocol $\mathrm{P}$ assigned to each state. Of course, a uniform protocol will be common knowledge among the agents (indeed, the same protocol is used at all states). On the other hand, state-dependent protocols are typically not known by any agents. Thus, state-dependent and uniform protocols are two extreme cases with many interesting cases in between, where agents have only partial knowledge of the type of conversation, experimental protocol, or learning process they are in. One natural example is the assumption that all agents individually know the protocol: for each $w, v \in D(\mathcal{M})$, if $w R_{i} v$ then $p(w)=p(v)$. For this paper, we will restrict attention to state-dependent protocols and uniform protocols.

We now turn to the main construction of this paper: generating an ETL model from an initial epistemic model and a (state-dependent or uniform) DEL protocol. We start with constructing and ETL model from a uniform DEL protocol since the definition is more transparent. However, we stress that the following two definitions are special cases of the more general construction given below (cf. Definition 2.10 and Definition 2.11).

\footnotetext{
${ }^{3}$ The preconditions of DEL also encode protocol information of a 'local' character, and hence they can do some of the work of global protocols, as has been pointed out in [28]. We do not pursue this division of labour here.
} 
Definition 2.8 ( $\sigma$-Generated Epistemic Model) Given a pointed epistemic model $\mathcal{M}, w$ and a finite sequence of pointed event models $\sigma$, we define the $\sigma$ generated epistemic model, $(\mathcal{M}, w)^{\sigma}$ as $(\mathcal{M}, w) \otimes \sigma_{(1)} \otimes \sigma_{(2)} \otimes \cdots \otimes \sigma_{(\operatorname{len}(\sigma))}$. We will write $\mathcal{M}^{\sigma}$ for $(\mathcal{M}, w)^{\sigma}$ when the state $w$ is clear from context.

\section{Definition 2.9 (ETL Model Generated from a Uniform DEL Protocol)} Let $\mathcal{M}$ be a pointed epistemic model, and P a DEL protocol. The ETL model generated by $\mathcal{M}$ and $\mathrm{P}$, Forest $(\mathcal{M}, \mathrm{P})$, represents all possible evolutions of the system obtained by updating $\mathcal{M}$ with sequences from $\mathrm{P}$. More precisely, Forest $(\mathcal{M}, \mathrm{P})=$ $\left\langle\Sigma, \mathrm{H},\left\{\sim_{i}\right\}_{i \in \mathcal{A}}, V\right\rangle$, where $\left\langle\mathrm{H},\left\{\sim_{i}\right\}_{i \in \mathcal{A}}, V\right\rangle$ is the union of all models of the form $\mathcal{M}^{\sigma}$ with $\sigma \in \mathrm{P}$.

Since any DEL protocol $\mathrm{P}$ is closed under prefixes, for any epistemic model $\mathcal{M}$, Forest $(\mathcal{M}, \mathrm{P})$ is indeed an ETL model. Here is a concrete illustration:

Example 1 (ETL model generated from a uniform DEL protocol): We illustrate the above construction in public announcement logic (PAL [23]) with each event model denoting an announcement or observation of some true formula. Let $\mathrm{P}=\{(P),(P, Q),(P, R)\}$ and consider the epistemic model depicted here:

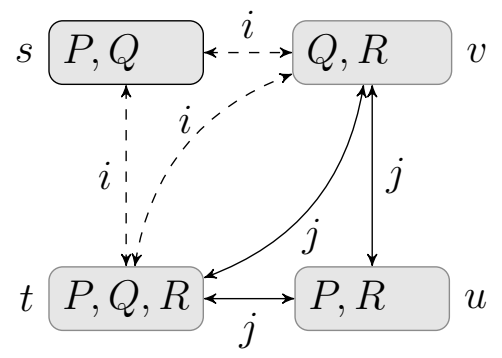

Using Definition 2.9, we can combine $\mathcal{M}$ and $\mathrm{P}$ to form an ETL model Forest $(\mathcal{M}, \mathrm{P})$ :

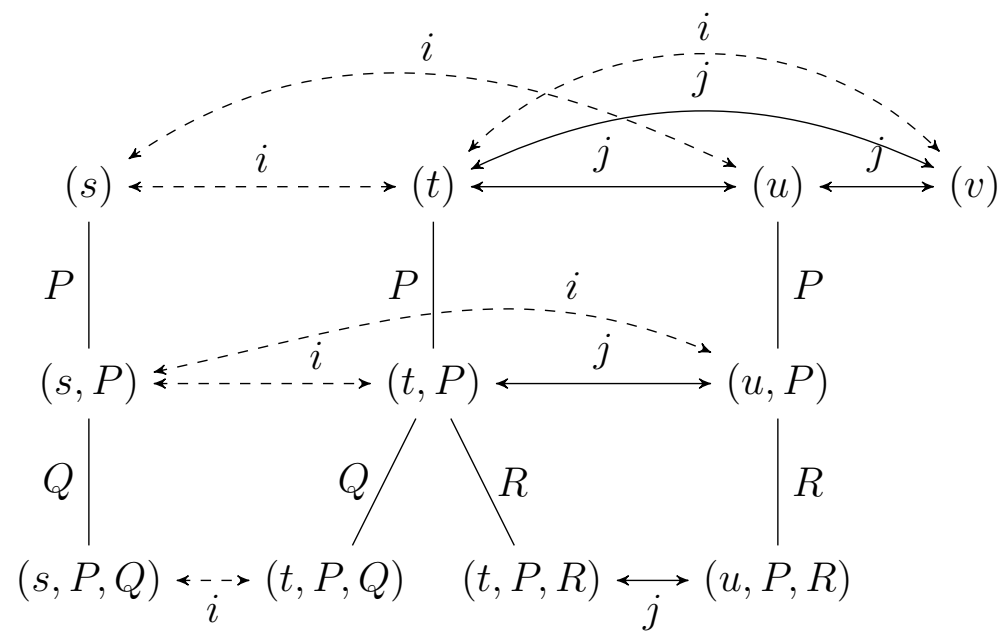


Note that in this example Forest $(\mathcal{M}, \mathrm{P}),(t) \models R \wedge \neg\langle R\rangle \top$. Thus even though a formula is true, it may not be "announcable" due to the underlying protocol. This reiterates the points raised in the Introduction and will be discussed in more detail in Section 4.1.2.

The ETL model Forest $(\mathcal{M}, \mathrm{P})$ in Example 1 satisfies a strong uniformity condition: if $(\mathcal{E}, e)$ is allowable according to the protocol $\mathrm{P}$, then for all histories $h$, the epistemic action $(\mathcal{E}, e)$ can be executed at $h$ iff pre $(e)$ is true at $h$. This implies that the protocol $\mathrm{P}$ is common knowledge ${ }^{4}$. Of course, this condition will not be satisfied in ETL models generated from state-dependent protocols.

Definition 2.10 ( $p$-Generated Model) Let $\mathcal{M}=\left\langle W,\left\{R_{i}\right\}_{i \in \mathcal{A}}, V\right\rangle$ be an epistemic model and $p$, a state-dependent DEL-protocol on $\mathcal{M}$. The $p$-generated model at level $n, \mathcal{M}^{n, p}=\left\langle W^{n, p},\left\{R_{i}^{n, p}\right\}_{i \in \mathcal{A}}, V^{n, p}\right\rangle$, is defined by induction on $n$ :

- $W^{0, p}=W$, for each $i \in \mathcal{A}, R_{i}^{0, p}=R_{i}$ and $V^{0, p}=V$.

- $w \sigma \in W^{n+1, p} \quad$ iff $(1) \quad w \in D(\mathcal{M}),(2) \operatorname{len}(\sigma)=n+1,(3) \quad w \sigma_{n} \in W^{n, p}$, (4) $\sigma \in p(w)$, and (5) $\mathcal{M}^{n, p}, w \sigma_{n} \models \operatorname{pre}\left(\sigma_{(n)}\right)$.

- For $w \sigma, v \sigma^{\prime} \in W^{n+1, p}, w \sigma R_{i}^{n+1, p} v \sigma^{\prime} \quad$ iff $\quad w \sigma_{n} R_{i}^{n, p} v \sigma_{n}^{\prime}$ and $\sigma_{(n+1)} \longrightarrow_{i}$ $\sigma_{(n+1)}^{\prime}$.

- For each $P \in$ At, $V^{n+1, p}(P)=\left\{w \sigma \in W^{n+1, p} \mid w \in V(P)\right\}$.

Definition 2.11 (Generated ETL Model) Let $\mathcal{M}=\left\langle W,\left\{R_{i}\right\}_{i \in \mathcal{A}}, V\right\rangle$ be an epistemic model and $p$ a state-dependent DEL protocol on $\mathcal{M}$. An ETL-model Forest $(\mathcal{M}, p)=\left\langle\mathrm{H},\left\{\sim_{i}\right\}_{i \in \mathcal{A}}, V^{\prime}\right\rangle$ is defined as follows:

- $\mathbf{H}=\left\{h \mid\right.$ there is a $w \in W, \sigma \in \bigcup_{w \in W} p(w)$ with $\left.h=w \sigma \in W^{\operatorname{len}(\sigma), p}\right\}$.

- For all $h, h^{\prime} \in \mathrm{H}$ with $h=w \sigma$ and $h^{\prime}=v \sigma^{\prime}, h \sim_{i} h^{\prime} \quad$ iff $\quad \operatorname{len}(\sigma)=\operatorname{len}\left(\sigma^{\prime}\right)$ and $w \sigma R_{i}^{\operatorname{len}(\sigma), p} v \sigma^{\prime}$.

- For each $P \in$ At and $h=w \sigma \in \mathrm{H}, h \in V^{\prime}(P) \quad$ iff $\quad h \in V^{\operatorname{len}(\sigma), p}(P)$. $\quad \triangleleft$

Since each DEL protocol $\mathrm{P}$ is closed under prefixes, so is the domain of Forest $(\mathcal{M}, p)$. Hence, Definition 2.11 indeed describes an ETL model. It is not difficult to see that Definition 2.8 and Definition 2.9 are special cases of Definiton 2.10 and Definition 2.11, respectively, when we restrict attention to uniform protocols (the details

\footnotetext{
${ }^{4}$ In fact, it implies the stronger fact that, if $(\mathcal{E}, e)$ can be executed, it can be executed anywhere in the current model (not just in the reachable states) provided pre $(e)$ is true.
} 
are left to the reader). We illustrate this construction with another example.

Example 2 (Making an ETL model from a state-dependent DEL protocol): Let $\mathcal{M}$ consist of two worlds, $w$ and $v$ which are indistinguishable for agent $i$ (the only one here). Furthermore, let the valuation make $P$ and $R$ true at both worlds and $Q$ true only at $w$. Let $p$ be a state-dependent DEL protocol defined as follows: $p(w)=\{(P),(P Q),(R)\}$ and $p(v)=\{(P),(P Q)\}$. Using Definition 2.11, we can combine $\mathcal{M}$ and $p$ to form an ETL model Forest $(\mathcal{M}, p)$ :

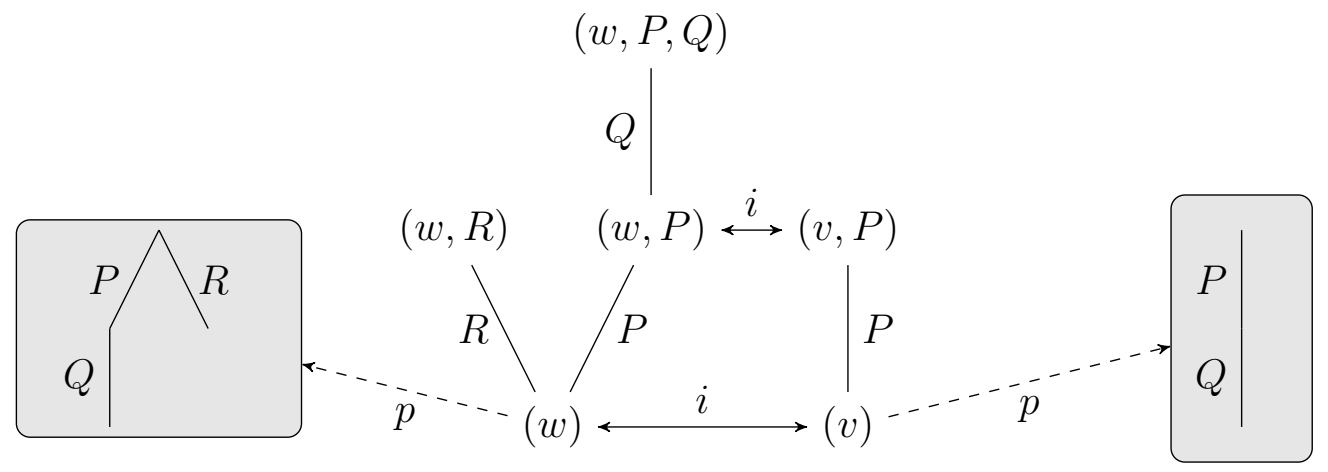

where the horizontal lines represent the indistinguishability relation and the dashed lines represent the state-dependent protocol function $p$. Note that this model does not satisfy the uniformity condition mentioned above. In fact, we have Forest $(\mathcal{M}, p),(w) \models\langle R\rangle \top$, but Forest $(\mathcal{M}, p),(v) \models R \wedge \neg\langle R\rangle \top$.

Our subsequent analysis will focus on two classes of structures. Given a class of state-dependent (or uniform) DEL protocols $\mathbf{X}$, let

$$
\mathbb{F}(\mathbf{X})=\{\text { Forest }(\mathcal{M}, p) \mid \mathcal{M} \text { an epistemic model and } p \in \mathbf{X}\}
$$

(respectively $\mathbb{F}(\mathbf{X})=\{$ Forest $(\mathcal{M}, \mathrm{P}) \mid \mathcal{M}$ an epistemic model and $\mathrm{P} \in \mathbf{X}\}$, when $\mathbf{X}$ is a set of uniform protocols). If $\mathbf{X}=\{p\}$ (respectively $\mathbf{X}=\{\mathrm{P}\}$ ) then we write $\mathbb{F}(p)$ (respectively $\mathbb{F}(\mathrm{P})$ ) instead of $\mathbb{F}(\{p\})$ (respectively $\mathbb{F}(\{\mathrm{P}\})$ ).

Our first observation is that under mild assumptions we can think of the languages $\mathcal{L}_{D E L}$ and $\mathcal{L}_{E T L}$ (when based on the same set of events $\Sigma$ ) as the same formal language. That is, the above model transformation allows use to reinterpret the DEL dynamic modality $\langle\mathcal{E}, e\rangle$ as a labeled temporal modality. Of course, to recover a DEL modality from an ETL temporal modality $\langle e\rangle$ we must know which event model $e$ belongs. The main point is that, since an primitive event $e$ may occur in different event models, a formula of $\mathcal{L}_{E T L}$ does not contain enough information to determine which event model different occurrences of the same primitive event $e$ belongs. However, once an ETL model is fixed this information 
can be extracted (see the proof of Theorem 3.7). Thus $\mathcal{L}_{E T L}$ and $\mathcal{L}_{D E L}$ are the same formal language under the mild assumption that it can always be determined which event models different occurrences of the same primitive event belongs.

An easy induction shows that this model transformation preserves truth in the following sense. Let ProtocolDEL be the protocol of all finite sequences of DEL event models and $\mathcal{M}$ an epistemic model with $w \in D(\mathcal{M})$ (and hence $(w)$ is a history in Forest $(\mathcal{M}$, ProtocolDEL $))$ :

Proposition 2.12 For any formula $\varphi \in \mathcal{L}_{D E L}$,

$$
\mathcal{M}, w \models \varphi \text { iff Forest }(\mathcal{M} \text {, ProtocolDEL }),(w) \models \varphi \text {. }
$$

Proposition 2.12 explains a common intuition about linking DEL to ETL. But there is more to come! Indeed, varying the parameters in Proposition 2.12 opens the door to a number of new questions. For example, we can extend the DEL language with temporal operators, or vary the protocol to create new DEL and ETL-style logics: much more on this will be found in Section 4 below.

\section{Connecting DEL and ETL}

Not all ETL models can be generated by a DEL protocol. Indeed, such generated ETL models have a number of special properties. In this section we study precisely which properties these are. The main result (Theorem 3.7) of this section is a characterization of the ETL models that are generated by some (uniform) DEL protocol. This is an improvement of an existing characterization result found in [28] and provides a precise comparison between the DEL and ETL frameworks.

We start with the result from Van Benthem [28] which characterizes the ETL models resulting from consecutive updates with one single event model. The following properties come from the definition of product update (Definition 2.5).

Definition 3.1 (Synchronicity, Perfect Recall, Uniform No Miracles) Let $\mathcal{H}=\left\langle\Sigma, H,\left\{\sim_{i}\right\}_{i \in \mathcal{A}}, V\right\rangle$ be an ETL model. $\mathcal{H}$ satisfies:

- Sychronicity iff for all $h, h^{\prime} \in \mathbf{H}$, if $h \sim_{i} h^{\prime}$ then $\operatorname{len}(h)=\operatorname{len}\left(h^{\prime}\right)(\operatorname{len}(h)$ is the number of events in $h$ ).

- Perfect Recall iff for all $h, h^{\prime} \in \mathrm{H}, e, e^{\prime} \in \Sigma$ with $h e, h^{\prime} e^{\prime} \in \mathbf{H}$, if $h e \sim_{i} h^{\prime} e^{\prime}$, then $h \sim_{i} h^{\prime}$

- Uniform No Miracles iff for all $h, h^{\prime} \in \mathbf{H}, e, e^{\prime} \in \Sigma$ with $h e, h^{\prime} e^{\prime} \in \mathbf{H}$, if there are $h^{\prime \prime}, h^{\prime \prime \prime} \in \mathrm{H}$ with $h^{\prime \prime} e, h^{\prime \prime \prime} e^{\prime} \in \mathrm{H}$ such that $h^{\prime \prime} e \sim_{i} h^{\prime \prime \prime} e^{\prime}$ and $h \sim_{i} h^{\prime}$, then $h e \sim_{i} h^{\prime} e^{\prime}$. 
Additional properties vary depending on the class of DEL protocols considered.

Remark 3.2 (Alternative Definition of Perfect Recall) Van Benthem gives an alternative definition of Perfect Recall in [28]:

if he $\sim_{i} h^{\prime}$ then there is an event $f$ with $h^{\prime}=h^{\prime \prime} f$ and $h \sim_{i} h^{\prime \prime}$.

This property is equivalent over the class of ETL models to the above definition of Perfect Recall and synchronicity. We use the above formulation of Perfect Recall in order to stay closer to the computer science literature on verifying multiagent systems (cf. [8]) and the game theory literature (cf. [7]).

The next property reflects that preconditions of events are formulas of $\mathcal{L}_{E L}$.

Definition 3.3 (Epistemic Bisimulation Invariance) Let $\mathcal{H}=\left\langle\mathrm{H},\left\{\sim_{i}\right\}_{i \in \mathcal{A}}, V\right\rangle$ and $\mathcal{H}^{\prime}=\left\langle\mathrm{H}^{\prime},\left\{\sim_{i}^{\prime}\right\}_{i \in \mathcal{A}}, V\right\rangle$ be two ETL models. A relation $Z \subseteq \mathrm{H} \times \mathrm{H}^{\prime}$ is an epistemic bisimulation provided that, for all $h \in \mathrm{H}$ and $h^{\prime} \in \mathrm{H}^{\prime}$, if $h Z h^{\prime}$, then

(prop) $h$ and $h^{\prime}$ satisfy the same propositional formulas,

(forth) for every $g \in \mathrm{H}$, if $h \sim_{i} g$ then there exists $g^{\prime} \in \mathrm{H}^{\prime}$ with $h^{\prime} \sim_{i} g^{\prime}$ and $g Z g^{\prime}$

(back) for every $g^{\prime} \in \mathrm{H}^{\prime}$, if $h^{\prime} \sim_{i}^{\prime} g^{\prime}$ then there exists $g \in \mathrm{H}$ with $h \sim_{i} g$ and $g Z g^{\prime}$.

If $Z$ is an epistemic bisimulation and $h Z h^{\prime}$ then we say $h$ and $h^{\prime}$ are epistemically bisimilar. An ETL model $\mathcal{H}$ satisfies epistemic bisimulation invariance iff for all epistemically bisimilar histories $h, h^{\prime} \in \mathrm{H}$, if $h e \in \mathrm{H}$ then $h^{\prime} e \in \mathrm{H} . \quad \triangleleft$

One final assumption is needed since we are assuming that product update does not change the ground facts. An ETL model $\mathcal{H}$ satisfies propositional stability provided for all histories $h$ in $\mathcal{H}$, events $e$ with $h e$ in $\mathcal{H}$ and all propositional variables $P$, if $P$ is true at $h$ then $P$ is true at $h e$. We remark that this property is not crucial for the results in this section and can be dropped provided we allow product update to change the ground facts (cf. [33]). Let $\mathcal{E}$ be a fixed event model and $\mathrm{P}_{\mathcal{E}}$ be the protocol that consists of all finite sequences of the repetition of $\mathcal{E}$. That is, $\mathrm{P}_{\mathcal{E}}=(\{(\mathcal{E}, e) \mid e \in D(\mathcal{E})\})^{*}-\{\lambda\}$, where $\lambda$ is the empty string.

Proposition 3.4 (van Benthem [28]) An ETL model $\mathcal{H}$ is of the form $\operatorname{Forest}\left(\mathcal{M}, \mathrm{P}_{\mathcal{E}}\right)$ for some epistemic model $\mathcal{M}$ and event model $\mathcal{E}$ iff $\mathcal{H}$ satisfies propositional stability, synchronicity, perfect recall, uniform no miracles, as well as epistemic bisimulation invariance. 
We do not repeat the proof from [28] here since it is a specific case of our main representation theorem (Theorem 3.7) given below. But there are many further DEL protocols of interest ${ }^{5}$. For example, let $\mathbf{X}_{P A L}^{u n i}$ be the class of all uniform $P A L$ protocols (a PAL protocol is a DEL protocol where each event model is a public announcement event model $)$ and recall that $\mathbb{F}\left(\mathbf{X}_{P A L}^{u n i}\right)=\{\operatorname{Forest}(\mathcal{M}, \mathrm{P}) \mid$ $\mathcal{M}$ an epistemic model and $\mathrm{P}$ a PAL protocol $\}$.

Proposition 3.5 (PAL-generated models) An ETL model $\left\langle\Sigma, \mathrm{H},\left\{\sim_{i}\right\}_{i \in \mathcal{A}}, V\right\rangle$ is in $\mathbb{F}\left(\mathbf{X}_{P A L}^{u n i}\right)$ iff it satisfies the minimal properties of Theorem 3.7, and:

- for all $h, h^{\prime}, h e, h^{\prime} e \in \mathrm{H}$, if $h \sim_{i} h^{\prime}$, then $h e \sim_{i} h^{\prime} e$ (all events are reflexive)

- for all $h, h^{\prime} \in \mathrm{H}$, if $h e \sim_{i} h^{\prime} e^{\prime}$, then $e=e^{\prime}$ (no different events are linked).

Again, the proof will be an easy variant of our first main new result in this paper: a characterization of the class of all DEL generated models. It turns out that we can use "local" versions the bisimulation invariance and no miracles properties.

Definition 3.6 (Local No Miracles, Local Bisimulation Invariance) Let $\mathcal{H}$ $=\left\langle\Sigma, \mathrm{H},\left\{\sim_{i}\right\}_{i \in \mathcal{A}}, V\right\rangle$ be an ETL model. $\mathcal{H}$ satisfies:

- Local No Miracles iff for all $h_{1}, h_{2}, h, h^{\prime} \in \mathbf{H}, e, e^{\prime} \in \Sigma$ with $h_{1} e, h_{2} e^{\prime} \in \mathbf{H}$, if $h_{1} e \sim_{i} h_{2} e^{\prime}$ and $h \sim_{i} h^{\prime}$ and $h_{1} \sim^{*} h$, then $h e \sim_{i} h^{\prime} e^{\prime}$ (provided $h e, h^{\prime} e^{\prime} \in \mathrm{H}$ ) (Here, $\sim^{*}$ is the reflexive transitive closure of the union of the $\sim_{i}$ relations.)

- Local Bisimulation Invariance iff for all $h, h^{\prime} \in \mathrm{H}$, if $h \sim^{*} h^{\prime}$ and $h$ and $h^{\prime}$ are epistemically bisimilar, and $h e \in \mathrm{H}$, then $h^{\prime} e \in \mathrm{H}$

Before proving the first main result of this paper, a few technical comments are in order. The following proof will construct a DEL protocol from an ETL model satisfying certain properties. In particular, an event model will be constructed at each level of a given ETL model. Therefore, at each level of the ETL model we will need to specify a formula of $\mathcal{L}_{E L}$ as a pre-condition for each primitive event $e$ (cf. Definition 2.5). Thus, we already see the role that local bisimulation invariance will play in the proof: without it, there is no hope of finding a formula of $\mathcal{L}_{E L}$ for a pre-condition of an event $e$. However, as is well-known, bisimulation-invariance alone is typically not enough to guarantee the existence of such a formula. More specifically, there are examples of infinite sets that are bisimulation closed but not definable by any formula of $\mathcal{L}_{E L}$ (however, it will be definable by a formula of epistemic logic with infinitary conjunctions - see [6] for a discussion). Thus, if the

\footnotetext{
${ }^{5}$ Van Benthem \& Liu [30] suggest that iterating one large disjoint union of event model involving suitable preconditions can 'mimic' ETL style evolution for more complex protocols with varying event models. We do not pursue this claim here.
} 
set of histories at some level in which an event $e$ can be executed is infinite, there may not be a formula of $\mathcal{L}_{E L}$ that defines this set to be used as a pre-condition for $e$. Such a formula will exist under an appropriate finiteness assumption: at each level there are only finitely many histories in which $e$ can be executed, i.e., for each $n$, the set $\{h \mid h e \in \mathrm{H}$ and $\operatorname{len}(h)=n\}$ is finite.

Theorem 3.7 (Main Representation Theorem) Let $\mathbf{X}_{D E L}^{u n i}$ be the class of uniform DEL protocols. If an ETL model is in $\mathbb{F}\left(\mathbf{X}_{D E L}^{u n i}\right)$ then it satisfies propositional stability, synchronicity, perfect recall, local no miracles, as well as local bisimulation invariance.

If an ETL model $\mathcal{H}$ satisfies the finiteness assumption, propositional stability, synchronicity, perfect recall, local no miracles, and local bisimulation invariance, then $\mathcal{H} \in \mathbb{F}\left(\mathbf{X}_{D E L}^{u n i}\right)$.

Proof. Suppose that $\mathcal{H}=\left\langle\Sigma, \mathrm{H},\left\{\sim_{i}\right\}_{i \in \mathcal{A}}, V\right\rangle \in \mathbb{F}\left(\mathbf{X}_{D E L}^{\text {uni }}\right)$. Then $\mathcal{H}=\operatorname{Forest}(\mathcal{M}, \mathrm{P})$ for some initial epistemic model $\mathcal{M}$ and DEL protocol $\mathrm{P}$. We show that $\mathcal{H}$ satisfies local bisimulation invariance, and leave it to the reader to check that $\mathcal{H}$ satisfies the remaining properties. Suppose that $h, h^{\prime} \in \mathrm{H}$ with $h \sim^{*} h^{\prime}, h$ and $h^{\prime}$ are epistemically bisimilar, and $h e \in \mathrm{H}$ for some event $e \in \Sigma(=D(\mathrm{P}))$. We must show $h^{\prime} e \in \mathrm{H}$. By construction (Definition 2.9), $h=s e_{1} e_{2} \cdots e_{n} e \in$ $D\left(\mathcal{M} \otimes \mathcal{E}_{1} \otimes \cdots \mathcal{E}_{n} \otimes \mathcal{E}\right)$ where $\left(\mathcal{E}_{1}, e_{1}\right)\left(\mathcal{E}_{2}, e_{2}\right) \cdots\left(\mathcal{E}_{n}, e_{n}\right)(\mathcal{E}, e) \in \mathrm{P}, s \in D(\mathcal{M})$, for each $i=1, \ldots, n, e_{i} \in D\left(\mathcal{E}_{i}\right)$ and $e \in D(\mathcal{E})$. In order to prove $h^{\prime} e \in \mathrm{H}$, it is enough to show $h^{\prime} e \in D\left(\mathcal{M} \otimes \mathcal{E}_{1} \otimes \cdots \mathcal{E}_{n} \otimes \mathcal{E}\right)$. This follows from two facts: (1) $h^{\prime} \in D\left(M \otimes \mathcal{E}_{1} \otimes \cdots \otimes \mathcal{E}_{n}\right)$ and $(2) h^{\prime}=\operatorname{pre}(e)$. (2) follows from the fact that $h$ and $h^{\prime}$ are epistemically bisimilar and pre $(e)$ is assumed to be a formula of $\mathcal{L}_{E L}$. (1) follows from the assumption that $h \sim^{*} h^{\prime}$.

Suppose $\mathcal{H}=\left\langle\Sigma, \mathrm{H},\left\{\sim_{i}\right\}_{i \in \mathcal{A}}, V\right\rangle$ is an ETL model satisfying the above properties. We must show there is an epistemic model $\mathcal{M}_{\mathcal{H}}$ and a DEL protocol $\mathrm{P}_{\mathcal{H}}$ such that $\mathcal{H}=\operatorname{Forest}(\mathcal{M}, \mathrm{P})$. For the initial epistemic model, let $\mathcal{M}=\left\langle W,\left\{R_{i}\right\}_{i \in \mathcal{A}}, V^{\prime}\right\rangle$ with $W=\{h \mid \operatorname{len}(h)=1\}$, for $h, h^{\prime} \in W$, define $h R_{i} h^{\prime}$ provided $h \sim_{i} h^{\prime}$, and for each $p \in$ At, $V^{\prime}(p)=V(p) \cap W$.

Call a history $h \in \mathrm{H}$ maximal if there is no $h^{\prime} \in \mathrm{H}$ such that $h \prec h^{\prime}$. Now, for each maximal history $h \in \mathrm{H}$, define the closure of $h$, denoted $C(h)$, to be the the smallest set that contains all finite prefixes of $h$, and if $h^{\prime} \in C(h)$ and $h^{\prime} \sim^{*} h^{\prime \prime}$, then also $h^{\prime \prime} \in C(h)$. Note that by perfect recall, $C(h)$ is closed under finite prefixes and is completely connected with respect to the $\sim^{*}$ relation. It is easy to see that ${ }^{6} \mathrm{H}=\bigcup\{C(h) \mid h$ is a maximal history $\}$.

\footnotetext{
${ }^{6}$ Note that $C(h)$ only contains finite histories. According to Definition $2.1, \mathrm{H}$ only contains finite histories. This restriction is not crucial, however, and our result remains true without it.
} 
We define, for each maximal history $h \in \mathrm{H}$ and $j=1, \ldots$, len $(h)$, an event model $\mathcal{E}_{j}^{h}=\left\langle S_{j}^{h},\left\{\longrightarrow_{i}\right\}_{i \in \mathcal{A}}\right.$, pre $\rangle$ as follows:

1. $S_{j}^{h}=\left\{e \in \Sigma \mid\right.$ there is a history $h$ of length $j$ in $\mathrm{H}$ with $\left.h=h^{\prime} \cdot e\right\}$.

2. For each $e, e^{\prime} \in S_{j}^{h}$, define $e \longrightarrow_{i} e^{\prime}$ provided there are histories $h$ and $h^{\prime}$ of length $j$ ending in $e$ and $e^{\prime}$ respectively, such that $h \sim_{i} h^{\prime}$.

3. For each $e \in S_{j}^{h}$, let $\operatorname{pre}(e)$ be the formula that characterizes the set $\{h \mid$ $h e \in \mathrm{H}$ and $\operatorname{len}(h)=j\}$. Such a formula does exist, due to local bisimulation invariance and the finiteness assumption.

Finally, let $\mathrm{P}=\left\{(\mathcal{E})_{j}^{h} \mid h\right.$ is a maximal history in $\mathrm{H}$ and $\left.j \leq \operatorname{len}(h)\right\}$. Clearly, $\mathrm{P}$ is a DEL protocol and so is an element of $\mathbf{X}_{D E L}^{u n i}$. It is easy to see that Forest $(\mathcal{M}, \mathrm{P})$ and $\mathcal{H}$ have the same set of histories. All that remains is to prove that the epistemic relations are the same in $\mathcal{H}$ and Forest $(\mathcal{M}, \mathrm{P})$

Claim For each $h_{1}, h_{2} \in \mathrm{H}, h_{1} \sim_{i} h_{2}$ in $\mathcal{H}$ iff $h_{1} \sim_{i} h_{2}$ in Forest $(\mathcal{M}, \mathrm{P})$.

Proof of Claim. The proof is by induction on the length of $h$ and $h^{\prime}$ (which can be assumed to be the same by synchronicity). If $\operatorname{len}(h)=1$, the claim is immediate by the definition of $M$.

For the induction step, let $h_{1}=h \cdot e$ and $h_{2}=h^{\prime} \cdot e^{\prime}$. Suppose $h_{1} \sim_{i} h_{2}$ in $\mathcal{H}$. Then by perfect recall, $h \sim_{i} h^{\prime}$ in $\mathcal{H}$. So, by the induction hypothesis, $h \sim_{i} h^{\prime}$ in Forest $(\mathcal{M}, \mathrm{P})$ as well. By the definition given above, $e \longrightarrow_{i} e^{\prime}$ in the appropriate event model $\mathcal{E}_{j}^{h_{m}}$ for a maximal history $h_{m}$ and $j=\operatorname{len}\left(h_{1}\right)$. It follows by the definition of product update that $h_{1} \sim_{i} h_{2}$ in $\operatorname{Forest}(\mathcal{M}, \mathrm{P})$.

For the other direction, assume $h_{1} \sim_{i} h_{2}$ in $\operatorname{Forest}(\mathcal{M}, \mathrm{P})$. Then, by definition of product update, $h \sim_{i} h^{\prime}$ in Forest $(\mathcal{M}, \mathrm{P})$ and $e \longrightarrow_{i} e^{\prime}$ in the appropriate event model. By the way the event model is defined, there must be some $x$ and $x^{\prime}$ with $x \cdot e \sim_{i} x^{\prime} \cdot e^{\prime}$ in $\mathcal{H}$, and therefore, by local no miracles, also $h \cdot e \sim_{i} h^{\prime} \cdot e^{\prime}$ in $\mathcal{H}$. QED (of Claim)

An immediate consequence is that $\mathcal{H}$ and $\operatorname{Forest}(\mathcal{M}, \mathrm{P})$ are the same model. QED

This Theorem identifies the minimal properties that any DEL generated model must satisfy, and thus it describes exactly what type of agent is presupposed in the DEL framework. The proof generalises the one in van Benthem \& Liu [30], which is an immediate special case. The proof of the characterization of PAL (Proposition 3.5) is also a simple variant. The details are left to the reader.

Note that the finiteness assumption can be dropped at the expense of allowing preconditions to come from a more expressive language (specifically, infinitary epistemic logic). Alternatively, we can define the preconditions to be 
sets of histories (instead of formulas of some logical language). A possible compromise is to work with state-dependent protocols instead of uniform protocols. More precisely, in the above proof, we set the precondition of $e \in S_{j}^{h}$ to be $\top$, and define a local DEL-protocol $p$ so that, for all $w \in W, p(w)=\left\{(\mathcal{E})_{j}^{h} \mid\right.$ $h$ is a maximal history in $\mathrm{H}$ and $j \geq \operatorname{len}(h)\}$. Using this observation, we can argue in the same style as above to show the following representation theorem for state-dependent DEL protocols.

Theorem 3.8 Let $\mathrm{X}_{D E L}$ be the class of all state-dependent DEL-protocols. Then, an ETL-model is in $\mathbb{F}\left(\mathrm{X}_{D E L}\right)$ iff it satisfies propositional stability, synchronicity, perfect recall, and local no miracles.

\subsection{Towards a Correspondence Theory}

Our representation theorems suggest a more general correspondence theory ${ }^{7}$ relating natural properties of ETL frames to formulas in suitable modal languages. This section will present the beginings of such a theory which will, in turn, motivate extensions of the basic language $\mathcal{L}_{E T L}$ later on.

Of course, finding syntactic correspondents for the principles described above depends on how we express the conditions. Here we focus on three properties (we follow the convention that, whenever we write he, it is assumed that he is actually in the ETL frame). The ETL-frames $\mathcal{H}$ in our theorems satisfied:

1. Synchronicity: if $h \sim_{i} h^{\prime}$, then len $(h)=\operatorname{len}\left(h^{\prime}\right)$

2. Perfect Recall: if $h e \sim_{i} h^{\prime} f$, then $h \sim_{i} h^{\prime}$

3. Local No Miracles: if $h_{1} e \sim_{i} h_{2} f, h_{1} \sim^{*} h$ and $h \sim_{i} h^{\prime}$, then $h e \sim_{i} h^{\prime} f$.

First of all, we note that there is nothing mysterious or surprising about the modal principles that we will find corresponding to the above principles. We essentially find a general ETL version of the crucial DEL reduction axiom:

$$
\langle\mathcal{E}, e\rangle\left\langle\sim_{i}\right\rangle \varphi \leftrightarrow \operatorname{pre}(e) \wedge\left\langle\sim_{i}\right\rangle \bigvee_{\substack{e \underset{i f}{i} \mathcal{E} \\ \text { in }}}\langle\mathcal{E}, f\rangle \varphi
$$

which permutes the order of the dynamic and epistemic modalities. As we will see, essentially the left-to-right direction of the above formula corresponds to Perfect Recall and the right-to-left direction corresponds to the No Miracles property (see [9] for a related discussion in the context of products of modal logics). We now

\footnotetext{
${ }^{7}[25]$ discusses related correspondence issues but without our connection to DEL protocols.
} 
present correspondents to the above properties in appropriate extensions of the basic modal language $\mathcal{L}_{E T L}$.

Each of the observations below can be proven using a standard Sahlqvist argument (see [6], Section 3.6, for details). To apply such an analysis, a few special features of the current setting need to be noted: 1 . all events $e$ are deterministic (so, $\langle e\rangle \varphi \rightarrow[e] \varphi$ is valid), and 2 . looking backwards, there is at most one event. Finally, to simplify presentation, we sometimes let the relations $\sim_{i}$ satisfy certain properties (e.g., symmetry) - but this is just a convenience.

Synchronicity. This property suggests the addition of a new limited global modality: $\langle=\rangle \varphi$ which means " $\varphi$ is true at histories of the same length". Let $\mathcal{H}=\left\langle\Sigma, \mathrm{H},\left\{\sim_{i}\right\}_{i \in \mathcal{A}}, V\right\rangle$ be an ETL model, then define

$$
\mathcal{H}, h \models\langle=\rangle \varphi \text { iff there is a } h \in \mathrm{H} \text { with } \operatorname{len}(h)=\operatorname{len}\left(h^{\prime}\right) \text { and } \mathcal{H}, h^{\prime} \models \varphi
$$

The following fact is straightforward:

Observation 3.9 An ETL frame $\mathcal{H}$ satisifies synchronicity iff $\left\langle\sim_{i}\right\rangle \varphi \rightarrow\langle=\rangle \varphi$ is valid on $\mathcal{H}$.

Perfect Recall. This property suggests extending with language with a temporal "past" modality. Let $\mathcal{H}=\left\langle\Sigma, \mathrm{H},\left\{\sim_{i}\right\}_{i \in \mathcal{A}}, V\right\rangle$ be an ETL model, then define

$$
\mathcal{H}, h \models\left\langle e^{-}\right\rangle \varphi \text { iff there is a } h^{\prime} \in \mathrm{H} \text { with } h^{\prime} e=h \text { and } \mathcal{H}, h^{\prime} \models \varphi
$$

Then, a standard Salqhvist argument shows that

Observation 3.10 An ETL frame $\mathcal{H}$ satisfies Perfect Recall iff

$$
\langle e\rangle\left\langle\sim_{i}\right\rangle\left(\varphi \wedge\left\langle f^{-}\right\rangle \top\right) \rightarrow\left\langle\sim_{i}\right\rangle\langle f\rangle \varphi
$$

is valid on $\mathcal{H}$.

Note that we are crucially using the fact that if $\left\langle f^{-}\right\rangle \top$ is true at a history $h$ then there is a unique $h^{\prime}$ with $h^{\prime} f=h$. Recall that Remark 3.2 states a somewhat stronger version of perfect recall (equivalent over ETL models to synchronicity plus the above Perfect Recall property):

$$
\text { if } h e \sim_{i} h^{\prime} \text {, then there is an event } f \text { with } h^{\prime}=h^{\prime \prime} f \text { and } h \sim_{i} h^{\prime \prime}
$$

Again, it is not hard to see that the above property corresponds to the following modal principle (without any need for an additional 'synchronicity modality' $\langle=\rangle$ ):

$$
\langle e\rangle\left\langle\sim_{i}\right\rangle \varphi \rightarrow\left\langle\sim_{i}\right\rangle \bigvee_{f \text { any event }}\langle f\rangle \varphi
$$


Local No Miracles. This property involves quantification over histories accessibel via the "common-knowledge" relation $\sim^{*}$. This suggests added common knowledge to the language: Let $\mathcal{H}=\left\langle\Sigma, \mathrm{H},\left\{\sim_{i}\right\}_{i \in \mathcal{A}}, V\right\rangle$ be an ETL model, then

$$
\mathcal{H}, h \models\left\langle\sim^{*}\right\rangle \varphi \text { iff there is a } h^{\prime} \in \mathrm{H} \text { with } h \sim^{*} h^{\prime} \text { and } \mathcal{H}, h^{\prime} \models \varphi
$$

where $\sim^{*}$ is the reflexive transitive closure of the union of the $\sim_{i}$ relations. This is the existential dual of the standard common knowledge operator. The usual

Salqhvist analysis will not work here because of the common knowledge operator; however, following van Benthem's recent extension of a Salqhvist correspondence theory to modal fixed-point languages [27], we have:

Observation 3.11 An ETL frame $\mathcal{H}$ satisfies Local No Miracles iff

$$
\left(\langle e\rangle\left\langle\sim_{i}\right\rangle\left\langle f^{-}\right\rangle \top \wedge\left\langle\sim^{*}\right\rangle\left(\varphi \wedge\langle e\rangle \top \wedge\left\langle\sim_{i}\right\rangle\langle f\rangle \psi\right)\right) \rightarrow\left\langle\sim^{*}\right\rangle\left(\varphi \wedge\langle e\rangle\left\langle\sim_{i}\right\rangle \psi\right)
$$

is valid on $\mathcal{H}$.

Thus, we see that the DEL reduction axiom corresponds to natural ETL properties which are captured in relevant modal languages. We will return to these and further language extensions in Section 5.

\section{Merging DEL and ETL}

The representation theorems in Section 3 are one way of comparing and contrasting the DEL and ETL paradigms. But in this Section we turn to our second objective of this paper: to illustrate some new issues that arise when DEL and ETL are merged as a model of multi-agent interactive communication and learning.

This Section focuses on questions related to axiomatization and completeness. Each set of DEL protocols induces a class of ETL models: those genereated by an initial model and a protocol from the given set. Recall that if $\mathbf{X}$ is a set of DEL protocols, we define $\mathbb{F}(\mathbf{X})=\{$ Forest $(\mathcal{M}, \mathrm{P}) \mid \mathcal{M}$ an epistemic model and $\mathrm{P} \in \mathbf{X}\}$. This construction suggests the following natural questions:

- Which DEL protocols generate interesting ETL models?

- Which modal languages are most suitable to describe these models?

- Can we axiomatize interesting classes of DEL-generated ETL models? 
For some specific combinations of model classes and logical languages we already know the answers. For example, recall that ProtocolDEL is the set of all finite sequences of DEL event models - i.e., the forest of all possible DEL event structures. Then $\mathbb{F}(\{$ ProtocolDEL $\})=\{$ Forest $(\mathcal{M}$, ProtocolDEL $) \mid \mathcal{M}$ an epistemic model $\}$ is the set consisting of all DEL-generated ETL trees. Its logic (with respect to the language $\mathcal{L}_{D E L}$ ) can be axiomatized using the well-known reduction axioms: indeed this is the standard completeness theorem for DEL: cf. [4].

\subsection{Constrained Public Announcement Logic}

In order to illustrate the interesting new questions that arise when merging the DEL and ETL frameworks, we study in this section the logics of ETL models generated by PAL-protocols (DEL-protocols consisting only of public announcements). More precisely, let $\mathbf{X}_{P A L}$ and $\mathbf{X}_{P A L}^{u n i}$ be the set of state-dependent PAL protocols and uniform PAL-protocols respectively. We will present the logics of the classes $\mathbb{F}\left(\mathbf{X}_{P A L}\right)$ and $\mathbb{F}\left(\mathbf{X}_{P A L}^{u n i}\right)$. These classes can be thought of as representing the space of all "conversation scenarios" or "learning procedures". We first

axiomatize the class $\mathbb{F}\left(\mathbf{X}_{P A L}\right)$, and then turn to the uniform case $\mathbb{F}\left(\mathbf{X}_{P A L}^{u n i}\right)$ which will require us to extend our language somewhat.

\subsubsection{Notation and Simple Observations}

In the restricted setting of public announcements, we can simplify many of the definitions from Section 2. This section presents the notation and assumptions needed for the main results of this Section.

Public Announcement Protocols: A PAL-protocol is a set of sequences of formulas of $\mathcal{L}_{E L}$ closed under the initial segment relation. More formally, define $\operatorname{Ptcl}\left(\mathcal{L}_{E L}\right)=\left\{\mathrm{P} \mid \mathrm{P} \subseteq \mathcal{L}_{E L}^{*}\right.$ where $\mathrm{P}$ is closed under initial segments $\}$. Given an epistemic model $\mathcal{M}=\left\langle W,\left\{R_{i}\right\}_{i \in \mathcal{A}}, V\right\rangle$, a state-dependent PAL-protocol on $\mathcal{M}$ is a function $p: W \rightarrow \operatorname{Ptcl}\left(\mathcal{L}_{E L}\right)$.

The Language $\mathcal{L}_{T P A L}$ : Let $\mathcal{L}_{T P A L}$ be the fragment of $\mathcal{L}_{D E L}$ where all event models are restricted to public announcements. That is, $\mathcal{L}_{T P A L}$ extends $\mathcal{L}_{E L}$ with operators of the form $\langle A\rangle$ where $A \in \mathcal{L}_{E L}$. The dual operator $[A]$ is defined as usual $(\neg\langle A\rangle \neg)$. Furthermore, we will use the more suggestive $K_{i}$ instead of $[i]$.

Semantics: In the current setting, we can simplify many of the definitions from Section 2. Here, we present the definitions for ETL-models generated from statedependent PAL-protocols. The definition for uniform PAL-protocols should be considered as a specific case of the state-dependent case. First some notation. 
Given a sequence $\sigma=A_{1} \cdots A_{n}$ of elements of $\mathcal{L}_{E L}$, let $\sigma_{m}$ (with $m \leq n$ ) denote the initial segment of length $m$ and $\sigma_{(m)}$ the $m$ th component of $\sigma$. So, for example, $\sigma_{2}=A_{1} A_{2}$ and $\sigma_{(2)}=A_{2}$.

Definition 4.1 (cf. Definition 2.10) Let $\mathcal{M}=\left\langle W,\left\{R_{i}\right\}_{i \in \mathcal{A}}, V\right\rangle$ be an epistemic model, and $p$ a state-dependent PAL-protocol on $\mathcal{M}$. We define

$$
\mathcal{M}^{\sigma, p}=\left\langle W^{\sigma, p},\left\{R_{i}^{\sigma, p}\right\}_{i \in \mathcal{A}}, V^{\sigma, p}\right\rangle
$$

by induction on the length of $\sigma$ :

- $W^{\sigma_{0}, p}=W$, for each $i \in \mathcal{A}, R_{i}^{\sigma_{0}, p}=R_{i}$ and $V^{\sigma_{0}, p}=V$.

- $w \sigma_{m+1} \in W^{\sigma_{m+1}, p} \quad$ iff $\quad(1) w \in W,(2) \mathcal{M}^{\sigma_{m}, p}, w \sigma_{m} \models \varphi_{m+1}$, and also(3) $\sigma_{m+1} \in$ $p(w)$.

- For each $w \sigma_{m+1}, v \sigma_{m+1} \in W^{\sigma_{m+1}, p}, w \sigma_{m+1} R_{i}^{\sigma_{m+1}, p} v \sigma_{m+1} \quad$ iff $\quad w R_{i} v$.

- For each $P \in$ At, $V^{\sigma_{m+1}, p}(P)=\left\{w \sigma_{m+1} \in W^{\sigma_{m+1}, p} \mid w \in V(P)\right\}$.

Definition 4.2 (cf. Definition 2.11) Let $\mathcal{M}=\left\langle W,\left\{R_{i}\right\}_{i \in \mathcal{A}}, V\right\rangle$ be an epistemic model and $p$ a state-dependent PAL protocol on $\mathcal{M}$. An ETL-model Forest $(\mathcal{M}, p)=\left\langle\mathrm{H},\left\{\sim_{i}\right\}_{i \in \mathcal{A}}, V^{\prime}\right\rangle$ is defined as follows:

- $\mathbf{H}=\left\{h \mid h \in W^{\sigma, p}\right.$ for some $\left.\sigma \in \bigcup_{w \in W} p(w)\right\}$.

- For all $h, h^{\prime} \in \mathrm{H}$ with $h=w \sigma$ and $h^{\prime}=v \sigma$ for some $\sigma \in \bigcup_{w \in W} p(w)$, $h \sim_{i} h^{\prime} \quad$ iff $h R_{i}^{\sigma, p} h^{\prime}$.

- For each $P \in$ At, $h \in V^{\prime}(p)$ iff $h \in V^{\sigma, p}(p)$, where $h=w \sigma$ for some $\sigma \in \bigcup_{w \in W} p(w)$.

Truth of formulas of $\mathcal{L}_{T P A L}$ is defined as in Section 2. We repeat the definition here to make this Section self-contained.

Definition 4.3 (Truth) Let $\mathcal{H} \in \mathbb{F}\left(\mathrm{X}_{P A L}\right)$ with $\mathcal{H}=\operatorname{Forest}(\mathcal{M}, p)=\left\langle\mathrm{H},\left\{\sim_{i}\right.\right.$ \}$\left._{i \in \mathcal{A}}, V\right\rangle$. For a history $h=w \sigma \in \mathrm{H}$ with $w \in D(\mathcal{M})$, the truth of $\varphi \in \mathcal{L}_{T P A L}$ is inductively defined as follows:

$$
\begin{array}{lll}
\mathcal{H}, h=p & \text { iff } & h \in V(P) \quad \text { (with } P \in \text { At) } \\
\mathcal{H}, h=\neg \varphi & \text { iff } & \mathcal{H}, h \not \varphi \\
\mathcal{H}, h=\varphi \wedge \psi & \text { iff } & \mathcal{H}, h \models \varphi \text { and } \mathcal{H}, h \models \psi \\
\mathcal{H}, h=K_{i} \varphi & \text { iff } & \forall h^{\prime} \in \mathrm{H}, \text { if } h \sim_{i} h^{\prime} \text { then } \mathcal{H}, h^{\prime} \models \varphi \\
\mathcal{H}, h=\langle A\rangle \varphi & \text { iff } & h A \in \mathrm{H} \text { and } \mathcal{H}, h A \models \varphi
\end{array}
$$

Consistency, satisfiability, validity, and other basic notions are defined as usual. $\triangleleft$ 
Before proving the main result of this Section, we note a number of simple observations. First of all, evaluation of epistemic formulas only depends on the current 'stage' of successive announcements.

Observation 4.4 Let $\mathcal{F}=\operatorname{Forest}(\mathcal{M}, p)$. For $\varphi \in \mathcal{L}_{E L}$, for histories $h$ in Forest $(\mathcal{M}, p)$ with $h=w \sigma$ where $w \in D(\mathcal{M})$ and $\sigma \in \mathcal{L}_{E L}^{*}$,

$$
\mathcal{F}, h \sigma=\varphi \quad \text { iff } \mathcal{M}^{\sigma, p}, w \sigma \models \varphi
$$

Next we see that a formula $\varphi \in \mathcal{L}_{T P A L}$ can describe, at most, what is true after a sequence of announcements bounded in length by the depth of $\varphi$.

Definition 4.5 (Depth of a Formula) Suppose $\varphi \in \mathcal{L}_{T P A L}$. The depth of $\varphi$, denoted $d(\varphi)$, is defined as follows:

- $d(P)=0$ with $P \in$ At

- $d(\neg \varphi)=d(\varphi)$

- $d(\varphi \wedge \psi)=\max (d(\varphi), d(\psi))$

- $d\left(K_{i} \varphi\right)=d(\varphi)$

- $d(\langle A\rangle \varphi)=1+d(\varphi)$

This definition is lifted to a set $X \subseteq \mathcal{L}_{T P A L}$ of formulas as follows: $d(X)=$ $\max \{d(\varphi) \mid \varphi \in X\}$.

Given a protocol $p$ on $\mathcal{M}$ and a sequence $\sigma \in\left(\mathcal{L}_{E L}\right)^{*}$ with $\sigma \in p(w)$ for some $w \in D(\mathcal{M})$, we define a protocol $p_{k}^{\sigma<}$ on $\mathcal{M}^{\sigma, p}$ so that $p_{k}^{\sigma<}(w \sigma)=\{\tau$ | $\sigma \tau \in p(w)$ and len $(\tau) \leq k\}$ for all $w \sigma \in D\left(\mathcal{M}^{\sigma, p}\right)$. This family represents which sequences of formulas of length $k$ or less are announcable after $\sigma$. Also, we define $p^{\sigma<}(w \sigma)=\{\tau \mid \sigma \tau \in p(w)\}$ when not stating the upper bound. A straightforward induction gives the following result:

Observation 4.6 Let $\mathcal{M}$ be an epistemic model, $p$ a state-dependent protocol on $\mathcal{M}$. For all $w \in D(\mathcal{M})$ and $\sigma \in \bigcup_{w \in D(\mathcal{M})} p(w)$,

$$
\begin{aligned}
& \text { Forest }(\mathcal{M}, p), w \sigma \models \varphi \quad \text { iff } \operatorname{Forest}\left(\mathcal{M}^{\sigma, p}, p_{d(\varphi)}^{\sigma<}\right), w \sigma \models \varphi \\
& \text { Forest }(\mathcal{M}, p), w \sigma \models \varphi \quad \text { iff } \operatorname{Forest}\left(\mathcal{M}^{\sigma, p}, p^{\sigma<}\right), w \sigma \models \varphi
\end{aligned}
$$


Next, we show that the histories relevant to evaluate the truth of a given formula $\varphi \in \mathcal{L}_{T P A L}$ are the ones that contain its subformulas. Let $s u b^{a}(\varphi)$ be the set of subformulas of $\varphi$ that are in $\mathcal{L}_{E L}$. Given a state-dependent protocol $p$ on a model $\mathcal{M}$, for $w \in \mathcal{D}(\mathcal{M})$ define $(p(w))_{s u b^{a}(\varphi)}$ as follows:

$$
(p(w))_{s u b^{a}(\varphi)}=\left\{\sigma \in p(w) \mid \text { for each } A \text { in } \sigma, A \in s u b^{a}(\varphi)\right\} .
$$

This set represents announcable sequences of announcements at $w$ that only consist of subformulas of $\varphi$. Now we can show the following by an easy induction.

Observation 4.7 Suppose $\mathcal{M}$ is an epistemic model and $f$ and $g$ are two protocols on $\mathcal{M}$. Suppose $(f(v))_{\text {sub }^{a}(\varphi)}=(g(v))_{\text {sub }^{a}(\varphi)}$ for all $v \in D(\mathcal{M})$. Then for all $w \in D(\mathcal{M})$,

$$
\text { Forest }(\mathcal{M}, f), w \models \varphi \quad \text { iff } \operatorname{Forest}(\mathcal{M}, g), w \models \varphi \text {. }
$$

Finally we state the analogue of Proposition 2.12. Given a formula $\varphi \in \mathcal{L}_{T P A L}$ and an epistemic model $\mathcal{M}$, define $f_{\varphi}$ so that, for all $w \in D(\mathcal{M}), f_{\varphi}(w)=$ $\left\{A_{1} \cdots A_{k} \mid A_{i} \in \operatorname{sub}^{a}(\varphi)(1 \leq i \leq k)\right.$ for some $\left.k\right\}$. In the light of the above lemma, $f_{\varphi}$ represents the announcable sequences of $\mathcal{L}_{E L}$ formulas that are relevant to the truth value of $\varphi$. We can show by an easy induction that the generated ETL-model from $f_{\varphi}$ preserves the truth value of $\varphi$ in $P A L$ in the following sense.

Observation 4.8 Let $\varphi \in \mathcal{L}_{T P A L}$. Then

$$
\mathcal{M}, w \models \varphi \text { iff } \operatorname{Forest}\left(\mathcal{M}, f_{\varphi}\right), w \models \varphi \text {. }
$$

\subsubsection{Axiomatization of $\mathbb{F}\left(\mathrm{X}_{P A L}\right)$}

One distinguishing feature of TPAL is that the truth of $A$ is no longer equivalent to the availability of $A$ for assertion. This means that the usual reduction axioms of PAL are no longer valid. Thus, the standard axiomatization of PAL does not work for TPAL, and we have to redo the work.

Definition 4.9 (TPAL-Axioms) Let TPAL be the smallest set of formulas of $\mathcal{L}_{T P A L}$ that contains the following axiom schemes.

PC Propositional validities

$\mathrm{K}_{i} K_{i}(\varphi \rightarrow \psi) \rightarrow\left(K_{i} \varphi \rightarrow K_{i} \psi\right)$

$\mathrm{R} 1\langle A\rangle P \leftrightarrow\langle A\rangle \top \wedge P$

$\mathrm{R} 2\langle A\rangle \neg \varphi \leftrightarrow\langle A\rangle \top \wedge \neg\langle A\rangle \varphi$ 


$$
\begin{aligned}
& \mathrm{R} 3\langle A\rangle(\varphi \wedge \psi) \leftrightarrow\langle A\rangle \varphi \wedge\langle A\rangle \psi \\
& \mathrm{R} 4\langle A\rangle K_{i} \varphi \leftrightarrow\langle A\rangle \top \wedge K_{i}(\langle A\rangle \top \rightarrow\langle A\rangle \varphi) \\
& \mathrm{A} 1\langle A\rangle(\varphi \rightarrow \psi) \rightarrow(\langle A\rangle \varphi \rightarrow\langle A\rangle \psi) \\
& \mathrm{A} 2\langle A\rangle \top \rightarrow A
\end{aligned}
$$

Furthermore, TPAL is closed under $K_{i^{-}}$and $[A]$ - necessitation and modus ponens. We write $\vdash \varphi$ if $\varphi \in T P A L$.

Remark 4.10 Notice that TPAL does not satisfy uniform substitution. For one thing, axiom $R 1$ only applies to atomic propositions $P \in$ At. Furthermore, only formulas of $\mathcal{L}_{E L}$ can be announced. So, for example, $\langle\langle B\rangle \top\rangle P \leftrightarrow\langle\langle B\rangle \top\rangle \top \wedge P$ is not an instance of axiom $R 1$. We could actually lift this restriction somewhat without endangering our results, but will not do so here.

These axioms illustrate the mixture of factual and procedural truth, which drives conversations or processes of observation ${ }^{8}$. In $T P A L,\langle A\rangle \top$ means that $A$ is announceable. More precisely, $\langle A\rangle \top$ represents one temporal step in a generated ETL model Forest $(\mathcal{M}, p)$ for some initial model $\mathcal{M}$ and state-dependent protocol $p$. So, axiom A2 represents the procedural information that "only true formulas can be announced". The converse (which is derivable in PAL) is valid only on a specific protocol following the rule "if $A$ is true then it can be announced".

Before turning to the main result of this Section, we consider axiom R4 in more detail. Consider the following three variations of R4:

1. $\langle A\rangle K_{i} P \leftrightarrow A \wedge K_{i}\langle A\rangle P$

2. $\langle A\rangle K_{i} P \leftrightarrow\langle A\rangle \top \wedge K_{i}(A \rightarrow\langle A\rangle P)$

3. $\langle A\rangle K_{i} P \leftrightarrow\langle A\rangle \top \wedge K_{i}(\langle A\rangle \top \rightarrow\langle A\rangle P)$

Each of these axioms represent a different assumption about the underlying protocol and how that affects the agents' knowledge. The first is the usual PAL reduction axiom and assumes a specific protocol (which is common knowledge) where all true formulas are always available for announcement. The second (weaker) axiom is valid when there is a fixed protocol that is common knowledge (cf. Section ??). Finally, the third is an instance of R4 which adds a requirement that the agents must know which formulas are currently available for announcement.

Our goal in this Section is to prove the following Theorem:

\footnotetext{
${ }^{8}$ Similarly to how we set up TPAL, Lorini and Castelfranchi ([18]) re-define PAL as "either take the restricted model, or let it be undefined", in effect defining a PAL protocol. They do not give a completeness result, but do formulate laws similar to our TPAL axioms.
} 
Theorem 4.11 TPAL is sound and strongly complete with respect to the class of ETL models $\mathbb{F}\left(\mathbf{X}_{P A L}\right)$.

The proof is a variant of the standard Henkin construction ${ }^{9}$. We construct the canonical ETL-model from the set of TPAL maximal consistent sets (mcs). The main idea is that each mcs defines sequences of 'legal' public announcements which we use to define a canonical state-dependent protocol. We start by defining the set of legal histories and a function $\lambda_{n}$ that assigns maximally consistent sets to each node on a history.

Definition 4.12 (Legal Histories) Let $W_{0}$ be the set of all TPAL maximal consistent sets. We define $\lambda_{n}$ and $H_{n}(0 \leq n \leq d(\Sigma))$ are defined as follows:

- Set $H_{0}=W_{0}$, and for each $w \in H_{0}, \lambda_{0}(w)=w$.

- Let $H_{n+1}=\left\{h A \mid h \in H_{n}\right.$ and $\left.\langle A\rangle \top \in \lambda_{n}(h)\right\}$. For each $h=h^{\prime} A \in H_{n+1}$, define $\lambda_{n+1}(h)=\left\{\varphi \mid\langle A\rangle \varphi \in \lambda_{n}\left(h^{\prime}\right)\right\}$.

We first confirm that each map $\lambda_{n}$ is well-defined.

Lemma 4.13 For each $n \geq 0$, for each $\sigma \in H_{n}, \lambda_{n}(\sigma)$ is maximally consistent.

Proof. The proof is by induction on $n$. The case $n=0$ is by definition. Suppose that the statement holds for $H_{n}$ and $\lambda_{n}$. Suppose $\sigma \in H_{n+1}$ with $\sigma=\sigma^{\prime} A$. By the induction hypothesis, $\lambda_{n}\left(\sigma^{\prime}\right)$ is a maximally consistent set. Furthermore, by the construction of $H_{n+1},\langle A\rangle \top \in \lambda_{n}(\sigma)$. Therefore, $\lambda_{n+1}(\sigma) \neq \emptyset$. Let $\varphi \in \mathcal{L}_{T P A L}$. Since $\lambda_{n}\left(\sigma^{\prime}\right)$ is a maximally consistent set, either $\langle A\rangle \varphi \in \lambda_{n}\left(\sigma^{\prime}\right)$ or $\neg\langle A\rangle \varphi \in \lambda_{n}\left(\sigma^{\prime}\right)$. If $\langle A\rangle \varphi \in \lambda_{n}\left(\sigma^{\prime}\right), \varphi \in \lambda_{n+1}(\sigma)$ by construction. If $\neg\langle A\rangle \varphi \in \lambda_{n}\left(\sigma^{\prime}\right)$, by axiom R2, we have $\langle A\rangle \neg \varphi \in \lambda_{n}\left(\sigma^{\prime}\right)$. Thus, by construction, $\neg \varphi \in \lambda_{n+1}(\sigma)$. Thus, for all $\varphi \in \mathcal{L}_{T P A L}$, either $\varphi \in \lambda_{n+1}(\sigma)$ or $\neg \varphi \in \lambda_{n+1}(\sigma)$.

To show that $\lambda_{n+1}$ is consistent, assume toward contradiction that there are formulas $\varphi_{1}, \ldots, \varphi_{m} \in \lambda_{n+1}(\sigma)$ such that $\vdash \bigwedge_{i=1}^{m} \varphi \rightarrow \perp$. Using standard modal reasoning, $\vdash\langle A\rangle \top \rightarrow \bigvee_{i=1}^{m}\langle A\rangle \neg \varphi_{i}$. Since $\langle A\rangle \top \in \lambda_{n}\left(\sigma^{\prime}\right)$, we have $\bigvee_{i=1}^{m}\langle A\rangle \neg \varphi \in$ $\lambda_{n}\left(\sigma^{\prime}\right)$. And so, since $\lambda_{n}\left(\sigma^{\prime}\right)$ is a maximally consistent set, there is some $j$ with $1 \leq j \leq m$ and $\langle A\rangle \neg \varphi_{j} \in \lambda_{n}\left(\sigma^{\prime}\right)$. Using axioms $R 2$, we have $\neg\langle A\rangle \varphi_{j} \in \lambda_{n}\left(\sigma^{\prime}\right)$. By construction of $\lambda_{n+1}(\sigma)$ we have for each $i=1, \ldots, m,\langle A\rangle \varphi_{i} \in \lambda_{n}\left(\sigma^{\prime}\right)$. This contradicts the fact that $\lambda_{n}\left(\sigma^{\prime}\right)$ is consistent.

QED

\footnotetext{
${ }^{9}$ The usual completeness proofs for PAL and DEL reduce the DEL expressions to standard modal logic. This device is no longer available to us here in a straightforward manner, though TPAL does allow for some 'normal form reduction'. Accordingly, the completeness proof for TPAL in this Section uses a Henkin-style model - a method also used for DEL in [11].
} 
We now define a canonical ETL model $\mathcal{H}^{\text {can }}$. We start by defining $\mathcal{H}_{0}^{\text {can }}=$ $\left\langle H_{0},\left\{\sim_{i}^{0}\right\}_{i \in \mathcal{A}}, V^{0}\right\rangle$. For this, we use the usual definitions:

- For $w, v \in H_{0}$, let $w \sim_{i}^{0} v \quad$ iff $\left\{\varphi \mid K_{i} \varphi \in w\right\} \subseteq v$.

- For each $P \in$ At and $w \in H_{0}, P \in V^{0}(w)$ iff $P \in w$.

Definition 4.14 (Canonical Model) The canonical model $\mathcal{H}^{c a n}=\left\langle\mathrm{H}^{c a n},\left\{\sim_{i}^{c a n}\right.\right.$ \}$\left._{i \in \mathcal{A}}, V^{\text {can }}\right)$ is defined as follows:

- $\mathrm{H}^{\text {can }}=\bigcup_{i=0}^{\infty} H_{i}$.

- For each $h, h^{\prime} \in \mathrm{H}^{\text {can }}$ with $h=w \sigma$ and $h^{\prime}=w^{\prime} \sigma^{\prime}$, let $h \sim_{i}^{\text {can }} h^{\prime}$ iff (1) $\sigma=\sigma^{\prime}$ and $(2) w \sim_{i}^{0} v$.

- For every $P \in$ At and $h=w \sigma \in \mathrm{H}^{c a n}, w \sigma \in V^{c a n}(P)$ iff $w \in V^{0}(P)$. $\quad \triangleleft$

Given $h \in \mathrm{H}^{\text {can }}$ with $h=w A_{1} \cdots A_{n}$, we write $\lambda(h)$ for $\lambda_{n}(h)$. We now show that the canonical model $\mathcal{H}^{\text {can }}$ works as intended:

Lemma 4.15 (Truth Lemma) For every $\varphi \in \mathcal{L}_{T P A L}$, for each $h \in \mathrm{H}^{\text {can }}$,

$$
\varphi \in \lambda(h) \quad \text { iff } \quad \mathcal{H}^{\text {can }}, h \models \varphi .
$$

Proof. We show by induction on the structure of $\varphi \in \mathcal{L}_{T P A L}$ that for each $h \in \mathrm{H}^{c a n}, \varphi \in \lambda(h)$ iff $\mathcal{H}^{c a n}, h \models \varphi$. The base and the boolean cases are straightforward. For the knowledge modality, let $h \in \mathrm{H}^{c a n}$ with $h=w A_{1} \cdots A_{n}$ and assume $K_{i} \psi \in \lambda(h)$. Suppose $h^{\prime} \in \mathrm{H}^{\text {can }}$ with $h \sim_{i} h^{\prime}$. By construction of the canonical model, we know that $h^{\prime}=v A_{1} \cdots A_{n}$ for some $v \in H_{0}$ with $w \sim_{i}^{0} v$. By Definition 4.12, since $K_{i} \psi \in \lambda\left(w A_{1} \cdots A_{n}\right)$, we have $\left\langle A_{n}\right\rangle K_{i} \psi \in \lambda\left(w A_{1} \cdots A_{n-1}\right)$. Using Axiom $R 4$, we have $K_{i}\left(\left\langle A_{n}\right\rangle \top \rightarrow\left\langle A_{n}\right\rangle \psi\right) \in \lambda\left(w A_{1} \cdots A_{n-1}\right)$. Continuing this way, we have

$$
K_{i}\left(\left\langle A_{1}\right\rangle \top \rightarrow\left\langle A_{1}\right\rangle\left(\left\langle A_{2}\right\rangle \top \rightarrow\left\langle A_{2}\right\rangle\left(\cdots\left\langle A_{n-1}\right\rangle\left(\left\langle A_{n}\right\rangle \top \rightarrow\left\langle A_{n}\right\rangle \psi\right) \cdots\right)\right) \in w .\right.
$$

By Definition 4.14, since $h \sim_{i}^{c a n} h^{\prime}$, we have $w \sim_{i}^{0} v$. Hence,

$$
\left\langle A_{1}\right\rangle \top \rightarrow\left\langle A_{1}\right\rangle\left(\left\langle A_{2}\right\rangle \top \rightarrow\left\langle A_{2}\right\rangle\left(\cdots\left\langle A_{n-1}\right\rangle\left(\left\langle A_{n}\right\rangle \top \rightarrow\left\langle A_{n}\right\rangle \psi\right) \cdots\right) \in v .\right.
$$

Now note that

$$
\left\langle A_{1}\right\rangle \top \in \lambda(w),\left\langle A_{2}\right\rangle \top \in \lambda\left(w A_{1}\right), \ldots,\left\langle A_{n}\right\rangle \top \in \lambda\left(w A_{1} \ldots A_{n-1}\right) .
$$

Thus, we have

$$
\left\langle A_{2}\right\rangle \top \rightarrow\left\langle A_{2}\right\rangle\left(\cdots\left\langle A_{n-1}\right\rangle\left(\left\langle A_{n}\right\rangle \top \rightarrow\left\langle A_{n}\right\rangle \psi\right) \cdots\right) \in \lambda\left(v A_{1}\right)
$$




$$
\begin{gathered}
\left\langle A_{3}\right\rangle \top \rightarrow\left\langle A_{3}\right\rangle\left(\cdots\left\langle A_{n-1}\right\rangle\left(\left\langle A_{n}\right\rangle \top \rightarrow\left\langle A_{n}\right\rangle \psi\right) \cdots\right) \in \lambda\left(v A_{1} A_{2}\right) \\
\vdots \\
\left\langle A_{n}\right\rangle \psi \in \lambda\left(v A_{1} \cdots A_{n-1}\right)
\end{gathered}
$$

Therefore, $\psi \in \lambda\left(v A_{1} \cdots A_{n}\right)=\lambda\left(h^{\prime}\right)$. By the induction hypothesis, $\mathcal{H}^{c a n}, h^{\prime} \models \psi$. Therefore, $\mathcal{H}^{\text {can }}, h \models K_{i} \psi$, as desired.

For the other direction, let $h \in \mathrm{H}^{\text {can }}$ and assume $K_{i} \psi \notin \lambda(h)$. For simplicity, we let $h=w A$ with $w \in W_{0}$ and $A \in \mathcal{L}_{E L}$. The argument can easily be generalized to deal with the general case along the lines of the argument above. Since $\lambda(h)$ is a maximally consistent set, we have $\neg K_{i} \psi \in \lambda(h)$. Thus, by Definition 4.12 , $\langle A\rangle \neg K_{i} \psi \in \lambda(w)$. Using axiom $R 2, \neg\langle A\rangle K_{i} \psi \in \lambda(w)$; and so, by Axiom $R 4$, $\neg\langle A\rangle \top \vee \neg K_{i}(\langle A\rangle \top \rightarrow\langle A\rangle \psi) \in \lambda(w)$. Since $\langle A\rangle \top \in \lambda(w)$ by construction, it follows that $\neg K_{i}(\langle A\rangle \top \rightarrow\langle A\rangle \psi) \in \lambda(w)$. Now consider the set $v_{0}=\left\{\theta \mid K_{i} \theta \in\right.$ $\lambda(w)\} \cup\{\neg(\langle A\rangle \top \rightarrow\langle A\rangle \psi)\}$. We claim that this set is cons istent. Suppose not. Then, there are formulas $\theta_{1}, \ldots, \theta_{m}$ such that $\vdash \bigwedge_{j=1}^{m} \theta_{j} \rightarrow\langle A\rangle \top \rightarrow\langle A\rangle \psi$ and for $j=1, \ldots, m, K_{i} \theta_{j} \in \lambda(w)$. By standard modal reasoning, $\vdash \bigwedge_{j=1}^{m} K_{i} \theta_{j} \rightarrow$ $K_{i}(\langle A\rangle \top \rightarrow\langle A\rangle \psi)$. This implies that $K_{i}(\langle A\rangle \top \rightarrow\langle A\rangle \psi) \in \lambda(w)$. However, this contradicts the fact that $\neg K_{i}(\langle A\rangle \top \rightarrow\langle A\rangle \psi) \in \lambda(w)$, since $\lambda(w)$ is a maximally consistent set. Now using standard arguments (Lindenbaum's lemma), there exists a maximally consistent set $v$ with $v_{0} \subseteq v$. By the construction of $v$, we must have $w \sim_{i}^{0} v$ and thus $w A \sim_{i}^{c a n} v A$. Also, since $\neg(\langle A\rangle \top \rightarrow\langle A\rangle \psi) \in v$, we have $\langle A\rangle \top \in \lambda(v)$ and $\neg\langle A\rangle \psi \in \lambda(v)$. Therefore, by axiom $R 2,\langle A\rangle \neg \psi \in \lambda(v)$. Hence $\neg \psi \in \lambda(v A)$ and therefore $\psi \notin \lambda(v A)$. By the induction hypothesis, $\mathcal{H}^{c a n}, v A \not \forall \psi$. This implies $\mathcal{H}^{c a n}, w A \not \models K_{i} \psi$, as desired.

For the public announcement operator, assume that $\langle A\rangle \psi \in \lambda(h)$. Since $\langle A\rangle \top \in$ $\lambda(h)$ (for $\neg\langle A\rangle \top \in \lambda(h)$ makes $\lambda(h)$ inconsistent), $\psi \in \lambda(h A)$. By the induction hypothesis, we have $\mathcal{H}^{\text {can }}, h A \models \psi$, which implies $\mathcal{H}^{\text {can }}, h \models\langle A\rangle \psi$. For the other direction, assume $\mathcal{H}^{\text {can }}, h \models\langle A\rangle \psi$. Then, $\mathcal{H}^{\text {can }}, h A \models \psi$. By the induction hypothesis, we have $\psi \in \lambda(h A)$ and thus $\langle A\rangle \psi \in \lambda(h)$.

QED

All that remains is to show that canonical model $\mathcal{H}^{\text {can }}$ is in the class of intended models: i.e., it is an element of $\mathbb{F}\left(\mathbf{X}_{P A L}\right)$.

Lemma $4.16 \mathcal{H}^{\text {can }}$ is in $\mathbb{F}\left(\mathbf{X}_{P A L}\right)$. That is, there is an epistemic model $\mathcal{M}$ and state-dependent protocol $p$ on $\mathcal{M}$ such that $\mathcal{H}^{\text {can }}=\operatorname{Forest}(\mathcal{M}, p)$.

Proof. Let $\mathcal{M}_{\text {can }}=\left(W_{0},\left\{\sim_{i}^{0}\right\}_{i \in \mathcal{A}}, V^{0}\right)$ and define $p_{\text {can }}: W_{0} \rightarrow \mathcal{L}_{E L}^{*}$ so that $p_{\text {can }}(w)=\left\{\sigma \mid w \sigma \in \mathrm{H}^{c a n}\right\}$. Suppose that $\mathcal{H}^{p_{\text {can }}}=$ Forest $\left(\mathcal{M}_{\text {can }}, p_{\text {can }}\right)$. We claim that $\mathcal{H}^{\text {can }}$ and $\mathcal{H}^{p_{\text {can }}}$ are the same model. For this, it suffices to show that for all $w \in W_{0}$ and $\sigma \in\left(\mathcal{L}_{E L}\right)^{*}$ we have $w \sigma \in \mathrm{H}^{\text {can }}$ iff $w \sigma \in W^{\sigma, p_{c a n}}$ (cf. Definition 
4.1). For this implies $\mathrm{H}^{\text {can }}=\mathrm{H}^{p_{\text {can }}}$, where $\mathrm{H}^{p_{\text {can }}}$ is the domain of $\mathcal{H}^{p_{\text {can }}}$. Then, by inspecting Definition 4.2 and Definition 4.14, we see that $\mathcal{H}^{\text {can }}$ and $\mathcal{H}^{p_{\text {can }}}$ are the same model.

We show by induction on the length of $\sigma \in \mathcal{L}_{E L}^{*}$ that for any $w \in W_{0}, w \sigma \in \mathrm{H}^{\text {can }}$ iff $w \sigma \in W^{\sigma, p_{c a n}}$. The base case (len $\left.(\sigma)=0\right)$ is clear. Assume that the claim holds for all $\sigma$ with $\operatorname{len}(\sigma)=n$.

Given any $\sigma \in \mathcal{L}_{E L}^{*}$ with len $(\sigma)=n$, we first show by subinduction (on the structure of $A$ ) that, for all $A \in \mathcal{L}_{E L}, \mathcal{H}^{\text {can }}, w \sigma \models A$ iff $\mathcal{M}^{\sigma, p_{\text {can }}}, w \sigma \models A$. The base and boolean cases are straightforward. Suppose that $\mathcal{H}^{\text {can }}, w \sigma=K_{i} B$. We must show $\mathcal{M}^{\sigma, p_{\text {can }}}, w \sigma \models K_{i} B$. Let $v \sigma \in W^{\sigma, p_{\text {can }}}$ with $w \sigma \sim_{i}^{\sigma, p} v \sigma$. By the main induction hypothesis, we have both $v \sigma \in \mathrm{H}^{c a n}$ and $w \sigma \in W^{\sigma, p_{c a n}}$. By Definition 4.1, since $w \sigma \sim_{i}^{\sigma, p_{c a n}} v \sigma$, we have $w \sim_{i}^{0} v$. Thus by Definition 4.14,w $w \sim_{i}^{c a n}$ $v \sigma$. Hence, $\mathcal{H}^{c a n}, v \sigma \models B$. By the subinduction hypothesis, $\mathcal{M}^{\sigma, p_{c a n}}, v \sigma \models B$. Therefore, $\mathcal{M}^{\sigma, p_{\text {can }}}, w \sigma \models K_{i} B$.

Coming back to the main induction, assume $w \sigma A \in \mathrm{H}_{\text {can }}$. This implies that $\langle A\rangle \top \in \lambda(w \sigma)$. By the Truth Lemma, we have $\mathcal{H}^{c a n}, w \sigma \models\langle A\rangle \top$. This, together with axiom $A 2$, implies $\mathcal{H}^{c a n}, w \sigma \models A$. From the above subinduction, it follows that $\mathcal{M}^{\sigma, p_{\text {can }}}, w \sigma \models A$ (recall that $A \in \mathcal{L}_{E L}$ by definition). Thus, by the construction of $p_{c a n}$, we have $w \sigma A \in W^{\sigma A, p_{c a n}}$. This shows that if $w \sigma A \in \mathrm{H}^{c a n}$ then $w \sigma A \in W^{\sigma A, p_{c a n}}$. The other direction is similar. This completes the proof. QED

The proof of the completeness theorem (Theorem 4.11) follows from Lemma 4.15 and Lemma 4.16 using a standard argument. The details are left to the reader.

\subsubsection{Axiomatization of $\mathbb{F}\left(\mathrm{X}_{P A L}^{u n i}\right)$}

Now we will axiomatize the class $\mathbb{F}\left(\mathbf{X}_{P A L}^{\text {uni }}\right)$ of ETL-models generated from uniform PAL-protocols. For this, we extend the language $\mathcal{L}_{T P A L}$ with an existential modality. Let $E \varphi$ mean that " $\varphi$ is true at some history with the same sequence of announcements". We define this as follows. Let $\mathcal{H}$ be an ETL model generated by an epistemic model $\mathcal{M}=\left\langle W,\left\{R_{i}\right\}_{i \in \mathcal{A}}, V\right\rangle$ and a (state-dependent or uniform) PAL protocol. Let $w \in W$ and $\sigma$ a sequence of announcements with $w \sigma \in D(\mathcal{H})$. Then we interpret the existential modality as accessibility at the same tree level:

$$
\mathcal{H}, w \sigma \models E \varphi \text { iff } \exists v \in W \text { such that } v \sigma \in D(\mathcal{H}) \text { and } \mathcal{H}, v \sigma \models \varphi \text {. }
$$

This operator functions as an existential modality at each 'stage' of successive public announcements. The dual $U$ of $E$ is a universal modality in the same sense. We consider the extension TPAL ${ }^{E}$ of TPAL.

First let us remark that the introduction of this operator keeps the system of TPAL manageable. In fact, all the basic semantic results for TPAL can be 
obtained for TPAL ${ }^{E}$ when we extend the notion of protocols with respect to $\mathcal{L}_{E L}^{E}$, the extension of $\mathcal{L}_{E L}$ with $E$. Also, a complete axiomatization can be given in a similar way by adding the following axioms to TPAL:

$$
\begin{aligned}
& \mathrm{E} 1 E(\varphi \rightarrow \psi) \rightarrow(E \varphi \rightarrow E \psi) \\
& \mathrm{E} 2 \varphi \rightarrow E \varphi \\
& \mathrm{E} 3 \varphi \rightarrow A E \varphi \\
& \mathrm{E} 4 E E \varphi \rightarrow E \varphi \\
& \mathrm{E} 5 U \varphi \rightarrow K_{i} \varphi \\
& \mathrm{R} 5\langle A\rangle E \varphi \leftrightarrow\langle A\rangle \top \wedge E\langle A\rangle \top .
\end{aligned}
$$

R5 allows us to obtain the results corresponding to Lemma 4.15 and Lemma 4.16 with respect to uniform protocols. Axioms $E 1-5$ are the standard axiomatization of the existential modality.

We now axiomatize the class

$$
\mathbb{F}\left(\mathbf{X}_{P A L}^{u n i}\right)=\{\text { Forest }(\mathcal{M}, \mathrm{P}) \mid \mathcal{M} \text { an epistemic model and } \mathrm{P} \text { a PAL protocol }\}
$$

For this, we extend the logic $T P A L^{E}$ with the following axiom:

$$
\mathrm{Uni}\langle A\rangle \top \rightarrow U(A \rightarrow\langle A\rangle \top) \text {. }
$$

This axiom characterizes uniform protocols in the following sense. Let us say a state-dependent protocol $p$ on a given model $\mathcal{M}$ generates a uniform ETL-model if Forest $(\mathcal{M}, p)=\operatorname{Forest}(\mathcal{M}, \mathrm{P})$ for some uniform PAL-protocol $\mathrm{P}$.

Proposition 4.17 The axiom Uni is valid on a frame Forest $(\mathcal{M}, p)$ iff p generates a uniform ETL-model.

Proof. $(\Leftarrow)$ Assume that $p$ generates a uniform ETL-model $\mathcal{H}=\operatorname{Forest}(\mathcal{M}, p)$. Then there is some uniform protocol $\mathrm{P}$ such that $\mathcal{H}=$ Forest $(\mathcal{M}, \mathrm{P})$. Now suppose that $w \in D(\mathcal{M})$ and $\sigma \in\left(\mathcal{L}_{E L}^{E}\right)^{*}$. Assume that $\mathcal{H}, w \sigma \models\langle A\rangle \top$. Then, we have $w \sigma A \in D(\mathcal{H})$. This means that $\sigma A \in p(w)$. Since $p$ is uniform, there is some PAL-protocol P such that $\mathcal{H}=\operatorname{Forest}(\mathcal{M}, \mathrm{P})$. Therefore $\sigma A \in \mathrm{P}$. Now, let $v$ be an arbitrary state in $\mathcal{M}$. If $\mathcal{H}, v \sigma \models A$, then, since $\sigma A \in \mathrm{P}$, we have $v \sigma A \in D(\mathcal{H})$. Hence $\mathcal{H}, v \sigma \models\langle A\rangle \top$. Since $v$ was arbitrary, we have $\mathcal{H}, w \sigma \models U(A \rightarrow\langle A\rangle \top)$.

$(\Rightarrow)$ Assume that Uni is valid on an ETL-model $\mathcal{H}^{p}=\operatorname{Forest}(\mathcal{M}, p)$. Construct a protocol $\mathrm{P}=\left\{\sigma \mid w \sigma\right.$ is in $\mathcal{H}^{p}$ for some $\left.w \in D(\mathcal{M})\right\}$. Clearly, $\mathrm{P}$ is closed under prefixes, so is in fact a PAL protocol. We need to show that $\mathcal{H}^{p}=\operatorname{Forest}(\mathcal{M}, \mathrm{P})$. 
For this, it suffices to show that, for all $\sigma, \mathcal{M}^{\sigma, p}=\mathcal{M}^{\sigma, \mathrm{P}}$, equivalently (via definition) $W^{\sigma, p}=W^{\sigma, \mathrm{P}}$. The left-to-right inclusion is clear by the construction of $\mathrm{P}$. For the other direction, we use induction on the length of $\sigma$. For the base case, $\sigma$ is the empty sequence; and so, the inclusion clearly holds as $W^{\sigma, p}=W^{\sigma, \mathrm{P}}=D(\mathcal{M})$. For the inductive step, assume that $w \sigma A \in W^{\sigma A, \mathrm{P}}$. Then we have $\mathcal{M}^{\sigma, \mathrm{P}}, w \sigma \models A$. By the induction hypothesis, we have $\mathcal{M}^{\sigma, p}, w \sigma=A$. Since $A \in \mathcal{L}_{E L}^{E}$, it follows from Observation 4.4 that $\mathcal{H}^{p}, w \sigma \models A$. Note that by the construction of $\mathrm{P}$, there must be some $v \in D(\mathcal{M})$ such that $v \sigma A \in W^{\sigma, p}$. This implies that $\mathcal{H}^{p}, v \sigma \models\langle A\rangle \top$. Here, since Uni is valid in $\mathcal{H}^{p}$, we have $\mathcal{H}^{p}, v \sigma \models U(A \rightarrow\langle A\rangle \top)$. Thus, it follows that $\mathcal{H}^{p}, w \sigma \models A \rightarrow\langle A\rangle \top$. From the fact that $\mathcal{H}^{p}, w \sigma \models A$, we then have $\mathcal{H}^{p}, w \sigma \models\langle A\rangle \top$, which is equivalent to $w \sigma A \in D\left(\mathcal{H}^{p}\right)$, i.e., $w \sigma A \in W^{\sigma A, p}$, as desired.

QED

Let $T P A L^{U n i}$ be the extension of $T P A L^{E}$ with the axiom $U n i$. The following is an immediate conseqeunce of a suitable truth lemma analogous to Lemma 4.15 and the above proposition:

Corollary 4.18 TPAL Uni is sound and strongly complete with respect to $\mathbb{F}\left(\mathbf{X}_{P A L}^{u n i}\right)$.

Proof. The proof is similar to the one outlined in Section 4.1.2 (making use of the above proposition to show that the canonical model is generated by a uniform protocol). The details are left to the reader.

QED

\subsubsection{Embedding $P A L$ in $T P A L$}

The introduction of the operator $E$ allows us to obtain another interesting result. Note that the relation between the original public announcement logic PAL and our variant TPAL s not immediate. PAL has more valid principles, being about special 'full' protocols. But is it really stronger than TPAL? The right way of asking such questions is in terms of effective embeddability under translation, rather than plain inclusion.

Indeed, we will now show that $P A L$ can be faithfully embedded into $T P A L^{E}$. Suppose $\varphi$ is a (T)PAL formula. We write $\models_{P A L^{E}} \varphi$ if $\varphi$ is valid on all epistemic models $\mathcal{M}$ where truth is defined as in Definition 2.5 (all event models are public announcements) and the existential modality is interpreted as above. We write $\models_{T P A L^{E}} \varphi$ when truth is defined as in Definition 4.3 and above (for the existential modality). First, we need some notation. Given a formula $\varphi$, let $\operatorname{Ptcl}(\varphi)$ be the set of formulas of the form:

$$
U\left(A_{1} \rightarrow\left\langle A_{1}\right\rangle\left(A_{2} \rightarrow\left\langle A_{2}\right\rangle\left(\cdots\left\langle A_{k}\right\rangle\left(A_{k} \rightarrow\left\langle A_{k}\right\rangle \top\right) \cdots\right)\right)\right)
$$

where $A_{i} \in \operatorname{sub}^{a}(\varphi)(1 \leq i \leq k)$ and $1 \leq k \leq d(\varphi)$. 
The formulas in $\operatorname{Ptcl}(\varphi)$ state that the public announcements that are relevant to the truth value of $\varphi$ are all announceable at any node of a given ETL-model.

Theorem 4.19 For any formula $\varphi \in \mathcal{L}_{T P A L}$

$$
\models_{P A L^{E}} \varphi \text { iff } \models_{T P A L^{E}} \bigwedge \operatorname{Ptcl}(\varphi) \rightarrow \varphi .
$$

Proof. $(\Leftarrow)$ Suppose $\models_{T P A L^{E}} \wedge \operatorname{Ptcl}(\varphi) \rightarrow \varphi$. Then, for all epistemic models $\mathcal{M}$ and all $w \in D(\mathcal{M})$, we have Forest $\left(\mathcal{M}, p_{P A L}\right), w=_{T P A L^{E}} \wedge \operatorname{Ptcl}(\varphi) \rightarrow \varphi$, where $p_{P A L}$ is the state-dependent protocol where for all $w \in D(\mathcal{M}), p_{P A L}(w)=\{\sigma \mid$ $\left.\sigma \in\left(\mathcal{L}_{E L}^{E}\right)^{*}\right\}$. By Proposition 2.12, $\mathcal{M}, w \models_{P A L} \wedge \operatorname{Ptcl}(\varphi) \rightarrow \varphi$. Now, notice that each formula in $\operatorname{Ptcl}(\varphi)$ is a theorem of $P A L$. Hence, $\mathcal{M},\left.w\right|_{P A L^{E}} \varphi$. Here observe that every formula in $\operatorname{Ptcl}(\varphi)$ reduces to tautology in $P A L$. Thus, we have $\mathcal{M}, w=_{P A L} \varphi$. Since $\mathcal{M}$ and $w$ were arbitrary, we have $\models_{P A L^{E}} \varphi$.

$(\Rightarrow)$ Suppose $=_{T P A L^{E}} \wedge \operatorname{Ptcl}(\varphi) \rightarrow \varphi$. Let Forest $(\mathcal{M}, p)$ be an arbitrary PALgenerated ETL model. Fix $h$ in Forest $(\mathcal{M}, p)$ and assume Forest $(\mathcal{M}, p), h \models_{T P A L^{E}}$ $\bigwedge \operatorname{Ptcl}(\varphi)$. Note that $h=w \sigma$ where $w \in D(\mathcal{M})$ and $\sigma$ a sequence of formulas in $\mathcal{L}_{E L}^{E}$. Now consider the epistemic model $\mathcal{M}^{\sigma, p}$. Since $\varphi$ is $P A L^{E}$-valid, we have $\mathcal{M}^{\sigma, p}, w \sigma=\varphi$. By Observation 4.8, Forest $\left(\mathcal{M}^{\sigma, p}, p_{\varphi}\right), w \sigma \models \varphi$. We now show that Forest $(\mathcal{M}, p)$ contains the model Forest $\left(\mathcal{M}^{\sigma, p}, p_{\varphi}\right)$.

Claim If $h^{\prime}$ is in Forest $\left(\mathcal{M}^{\sigma, p}, p_{\varphi}\right)$, then $h^{\prime}$ is in Forest $(\mathcal{M}, p)$.

Proof of Claim. We prove this claim by induction on the length of $h^{\prime}(\operatorname{len}(h) \leq$ $\left.\operatorname{len}\left(h^{\prime}\right) \leq \operatorname{len}(h)+d(\varphi)\right)$. For the base case, assume that len $(h)=\operatorname{len}\left(h^{\prime}\right)$. If $h^{\prime} \in D\left(\right.$ Forest $\left.\left(\mathcal{M}^{\sigma, p}, p_{\varphi}\right)\right)$, then $h^{\prime} \in D\left(\mathcal{M}^{\sigma, p}\right)$. Thus, $h^{\prime} \in D($ Forest $(\mathcal{M}, p)$. For the inductive step, assume that $h^{\prime} \in D\left(\right.$ Forest $\left.\left(\mathcal{M}^{\sigma, p}, p_{\varphi}\right)\right)$. Then we have $h^{\prime}=$ $v \sigma A_{1} \ldots A_{n}$ for $A_{i} \in \operatorname{sub}^{a}(\varphi)(1 \leq i \leq n)$ and $v \in D(\mathcal{M})$. Here, our assumption that Forest $(\mathcal{M}, p), w \sigma \models \bigwedge \operatorname{Ptcl}(\varphi)$ implies

$$
\text { Forest }(\mathcal{M}, p), w \sigma \models_{T P A L^{E}} U\left(A_{1} \rightarrow\left\langle A_{1}\right\rangle\left(\cdots\left(A_{n} \rightarrow\left\langle A_{n}\right\rangle \top\right) \cdots\right)\right)
$$

and so,

$$
\text { Forest }(\mathcal{M}, p), v \sigma=A_{1} \rightarrow\left\langle A_{1}\right\rangle\left(\cdots\left(A_{n} \rightarrow\left\langle A_{n}\right\rangle \top\right) \cdots\right) .
$$

Also, we have assumed that $h^{\prime}$ is in Forest $\left(\mathcal{M}^{\sigma, p}, p_{\varphi}\right)$, whose construction implies that $\mathcal{M}^{\sigma, p}, v \sigma \models A_{1}, \ldots, \mathcal{M}^{\sigma A_{1} \ldots A_{n-1}, p}, v \sigma A_{1} \ldots A_{n-1} \models A_{n}$. Now by the induction hypothesis, $v \sigma, v \sigma A_{1}, \ldots, v \sigma A_{1} \ldots A_{n-1}$ are all in Forest $(\mathcal{M}, p)$. From this, it follows that Forest $(\mathcal{M}, p), v \sigma \models A_{1}, \ldots$, Forest $(\mathcal{M}, p), v \sigma A_{1} \ldots A_{n-1} \models A_{n}$ (because we had $\left.A_{1}, \ldots, A_{n} \in \mathcal{L}_{E L}^{E}\right)$.

Thus, we have

$$
\text { Forest }(\mathcal{M}, p), v \sigma=\left\langle A_{1}\right\rangle\left(A_{2} \rightarrow\left\langle A_{2}\right\rangle\left(\cdots\left(A_{n} \rightarrow\left\langle A_{n}\right\rangle \top\right) \cdots\right)\right.
$$




$$
\begin{gathered}
\text { Forest }(\mathcal{M}, p), v \sigma A_{1} \models\left\langle A_{2}\right\rangle\left(A_{3} \rightarrow\left\langle A_{3}\right\rangle\left(\cdots\left(A_{n} \rightarrow\left\langle A_{n}\right\rangle \top\right) \cdots\right)\right. \\
\vdots \\
\text { Forest }(\mathcal{M}, p), v \sigma A_{1} \cdots A_{n-1} \models A_{n} \rightarrow\left\langle A_{n}\right\rangle \top . \\
\quad \text { Forest }(\mathcal{M}, p), v \sigma A_{1} \cdots A_{n-1} \models\left\langle A_{n}\right\rangle \top .
\end{gathered}
$$

Therefore, $h^{\prime}=v \sigma A_{1} \ldots A_{n}$ is in Forest $(\mathcal{M}, p)$ QED (of Claim)

Now, by the preceding claim, Forest $(\mathcal{M}, p)$ includes Forest $\left(\mathcal{M}^{\sigma, p}, p_{\varphi}\right)$. Therefore, Forest $\left(\mathcal{M}^{\sigma, p}, p_{\varphi}\right), w \sigma \models \varphi$, and by Observations 4.6 and 4.7, this implies that Forest $(\mathcal{M}, p) \models \varphi$. This completes the proof.

QED

We do not know if we can do this reduction without the existential modalitiy. Also, we have not solved the opposite question, whether TPAL can be faithfully embedded into PAL, though we think the answer is negative.

\subsubsection{Decidability}

We now turn to the complexity of our general logic of protocols. Indeed, like for PAL, the satisfiability problem for TPAL is decidable.

We show this by constructing a finite model for a given satisfiable formula $\varphi$. The basic idea follows the full completeness proof from Section 4.1.2. We construct a canonical model from a finite set of formulas that satisfies certain closure conditions. The decidability of the logic for uniform protocols can probably be obtained in a similar way, but, at this point, we have not found the appropriate closure condition for the existential modality.

Definition 4.20 (TPAL-Closed Sets) Let $X$ be a set of TPAL formulas. $X$ is $T P A L$-closed if $X$ satisfies the following closure conditions:

1. Closed under subformulas: If $\varphi \in X$ and $\psi$ is a subformula of $\varphi$, then $\psi \in X$.

2. Closed under single negations: If $\varphi \in X$ and $\varphi$ is of the form $\neg \psi$, then $\psi \in X$; and if $\varphi \in X$ and $\varphi$ is not of the form $\neg \psi, \neg \varphi \in X$.

3. If $\langle A\rangle \varphi \in X$, then $\langle A\rangle \top \in X$.

4. If $\langle A\rangle K_{i} \varphi \in X$, then $K_{i}(\langle A\rangle \top \rightarrow\langle A\rangle \varphi) \in X$.

5. If $\varphi \in X$, then $\left\langle A_{1}\right\rangle \ldots\left\langle A_{k}\right\rangle \varphi \in X(1 \leq k \leq d(X)-d(\varphi))$ where $\left\langle A_{i}\right\rangle \top \in X$ for every $1 \leq i \leq k$. 
Given a set $X \subseteq \mathcal{L}_{T P A L}$, we denote by $(X)^{T P A L}$ the smallest expansion of $X$ that is TPAL-closed. Note that provided that $X$ is a finite set of formulas, $(X)^{T P A L}$ is also finite; also, $d(X)=d\left((X)^{T P A L}\right)$. We denote by $(X)_{k}^{T P A L}$ with $0 \leq k \leq d(X)$ the set $\left\{\varphi \in(X)^{T P A L} \mid d(\varphi) \leq k\right\}$.

Let $\Sigma$ be a set of TPAL formulas. We call a set $a \subseteq(\Sigma)_{k}^{T P A L}$ an atom of depth $k$ over $\Sigma(0 \leq k \leq d(\Sigma))$, if $a$ is TPAL-consistent and if $a \subset b \subseteq(\Sigma)_{k}^{T P A L}$, then $b$ is inconsistent. We denote the set of the atoms of depth $k$ over $\Sigma$ as $A t_{k}(\Sigma)$. Now it is easy to check the following properties of atoms.

Lemma 4.21 Let $\Sigma$ be a set of TPAL formulas. For every $a \in A t_{k}(\Sigma)$, the following properties hold:

1. For all $\varphi \in(\Sigma)_{k}^{T P A L}, \varphi \in$ a or $\neg \varphi \in a$, but not both.

2. For all $\varphi \wedge \psi \in(\Sigma)_{k}^{T P A L}, \varphi \wedge \psi \in a$ iff $\varphi \in a$ and $\psi \in a$.

3. For all $\langle A\rangle p \in(\Sigma)_{k}^{T P A L}$ with $p$ a proposition letter, $\langle A\rangle \varphi \in$ a iff $\langle A\rangle \top \in a$ and $p \in a$.

4. For all $\langle A\rangle \neg \varphi \in(\Sigma)_{k}^{T P A L},\langle A\rangle \neg \varphi \in a$ iff $\langle A\rangle \top \in a$ and $\neg\langle A\rangle \varphi \in a$.

5. For all $\langle A\rangle(\varphi \wedge \psi) \in(\Sigma)_{k}^{T P A L},\langle A\rangle(\varphi \wedge \psi) \in a$ iff $\langle A\rangle \varphi \in a$ and $\langle A\rangle \psi \in a$.

6. For all $\langle A\rangle K_{i} \varphi \in(\Sigma)_{k}^{T P A L},\langle A\rangle K_{i} \varphi \in a$ iff $\langle A\rangle \top \in$ a and $K_{i}(\langle A\rangle \top \rightarrow$ $\langle A\rangle \varphi) \in a$.

7. For all $\langle A\rangle \varphi \in(\Sigma)_{k}^{T P A L}$, if $\langle A\rangle \varphi \in a$, then $\langle A\rangle \top \in a$.

8. For all $\langle A\rangle \top \in(\Sigma)_{k}^{T P A L}$, if $\langle A\rangle \top \in a$, the $A \in a$.

Proof. Immediate from the definition of an atom and Definition 4.20. $\quad$ QED

Given a finite set $\Sigma$ of $T P A L$-formulas, we construct a finite canonical model from the set $(\Sigma)^{T P A L}$. The construction follows exactly the construction from Section 4.1.2 (cf. Definition 4.12 and Definition 4.14). First, as in Definition ?? we construct maps $\lambda_{n}^{\text {fin }}$ and sets $H_{n}^{\text {fin }}(0 \leq n \leq d(\Sigma))$ as follows:

- Let $H_{0}^{f i n}=A t_{d(\Sigma)}(\Sigma)$ and for each $a \in H_{0}^{f i n}, \lambda_{0}^{f i n}(a)=a$.

- Let $H_{n+1}^{f i n}=\left\{\sigma A \mid \sigma \in H_{n}^{f i n}\right.$ and $\left.\langle A\rangle \top \in \lambda_{n}^{f i n}(\sigma)\right\}$. For every $\sigma=\sigma^{\prime} A \in$ $H_{n+1}^{\text {fin }}$, define $\lambda_{n+1}^{\text {fin }}(\sigma)=\left\{\psi \mid\langle A\rangle \psi \in \lambda_{n}^{\text {fin }}\left(\sigma^{\prime}\right)\right\}$.

Proposition 4.22 For all $n, \lambda_{n}^{\text {fin }}(\sigma) \in A t_{d(\Sigma)-n}(\Sigma)$. 
Proof. The proof is by induction on $n$. The base case is clear. For the inductive step, the argument is completely analogous to the proof of Lemma 4.13, given Lemma 4.21 and Definition 4.20.

QED

We now define a finite canonical model $\mathcal{H}^{\text {fin }}$. This goes exactly like Definition 4.14 except for the domain, which is now $H^{f} i n=\bigcup_{0 \leq i \leq d(\Sigma)} H_{i}^{f i n}$. As in Section 4.1.2, we write $\lambda^{\text {fin }}(h)$ from $\lambda_{n}^{f i n}(h)$ where $n$ is the number of announcements in $h$. We use $\sim_{i}^{f i n}$ and $V^{\text {fin }}$ to denote the canonical relations and valuations in $\mathcal{H}^{\text {fin }}$, just as in Definition 4.14. All that remains is prove are analogues of Lemma 4.15 and Lemma 4.16.

Lemma 4.23 (Finite Truth Lemma) Let $\varphi \in(\Sigma)^{T P A L}$. For every history $h$ in $H^{\text {fin }}$ such that len $(h) \leq d(\Sigma)-d(\varphi)+1$,

$$
\varphi \in \lambda^{\text {fin }}(h) \text { iff } \mathcal{H}^{\text {fin }}, h \models \varphi .
$$

Proof. The proof is by induction on $\varphi$. Given Lemma 4.21 and the closure conditions in Definition 4.20, the proof is similar to the proof of Lemma 4.15. We only present the public announcement modality case. Readers are invited to verify that the argument holds for the other cases as well. In particular, note that the formulas used in the proof of Lemma 4.15 are in fact in the set $\lambda^{\text {fin }}(h)$.

Let $\varphi$ be $\langle A\rangle \psi$. First, assume that $\langle A\rangle \psi \in \lambda^{\text {fin }}(h)$, where len $(h) \leq d(\Sigma)-$ $d(\varphi)+1$. Since $\langle A\rangle \psi \in \lambda^{\text {fin }}(h)$ by Lemma 4.20, $\langle A\rangle \top \in \lambda^{\text {fin }}(h)$. Thus, $h A \in H^{\text {fin }}$ and $\psi \in \lambda^{f i n}(h A)$. Here note that $\operatorname{len}(h A)=\operatorname{len}(h)+1 \leq d(\Sigma)-(d(\varphi)-1)+1=$ $d(\Sigma)-d(\psi)+1$. Thus, by induction, we have $\mathcal{H}^{\text {fin }}, h A=\psi$, which implies $\mathcal{H}^{\text {fin }}, h \models\langle A\rangle \psi$. For the other direction, assume that $\mathcal{H}^{\text {fin }}, h \models\langle A\rangle \psi$. This implies $\mathcal{H}^{\text {fin }}, h A=\psi$ with $\operatorname{len}(h A) \leq d(\Sigma)-d(\psi)+1$. By induction, $\psi \in \lambda^{\text {fin }}(h A)$. By the construction of the canonical model, $\langle A\rangle \psi \in \lambda^{f i n}(h)$ as desired. QED

Lemma $4.24 \mathcal{H}^{\text {fin }}$ is an ETL-model generated from an epistemic model and a PAL-protocol.

Proof. The proof is similar to that of Lemma 4.16 .

QED

Putting everything together, it is not difficult to verify that:

Theorem 4.25 (Decidability of TPAL) The satisfiability problem for the logic TPAL is decidable. 


\section{$5 \quad$ Language Extensions}

$\mathcal{L}_{E T L}$ is only one of many languages for reasoning about DEL generated ETL models, and there are many other temporal and epistemic operators of interest. We have explored some of these extensions in Section 3.1. Specifically, we made use of a common knowledge operator $C \varphi$ and a "backwards-looking" operator $\left\langle e^{-}\right\rangle \varphi$ meaning that $\varphi$ was true before event $e$ happened (and $e$ happened just before) to provide syntactic correspondents for various ETL frame-properties. Of course, other language extensions are worth exploring. For example, formulas of the form $F \varphi$ : " $\varphi$ is true some time in the future" or $\left\langle e^{*}\right\rangle \varphi$ : " $\varphi$ is true after a finite sequence of $e$ events" express the temporal future of the current process.

Extending the modal language with temporal and group modalities raises a number of interesting conceptual and technical issues. The next Section 6 higlights some that arise with a focus on specific types of DEL protocols. In the present Section, we point to some questions and results on axiomatization of classes of DEL generated ETL models in extensions of $\mathcal{L}_{E T L}$. Here we can profit from existing work on modal languages for reasoning about arbitrary ETL models, not just those generated by DEL protocols. The key results of Halpern and Vardi show that both imposing agent idealizations (such as Perfect Recall or No Miracles) and language extensions (arbitrary future and common knowledge) lead to high undecidability results [14]. Van Benthem and Pacuit [32] provide an overview of these results and related ones from other areas of computer science.

Group Knowledge Operators. We have already seen a formal definition of common knowledge in Section 3.1 when finding a syntactic correspondent to the local no miracles property. Let $\mathcal{H}=\left\langle\Sigma, \mathrm{H},\left\{\sim_{i}\right\}_{i \in \mathcal{A}}, V\right\rangle$ be an ETL model and recall that $\sim^{*}$ is the reflexive transitive closure of the relation $\bigcup_{i \in \mathcal{A}} \sim_{i}$. The common knowledge of $\varphi$ (denoted $C \varphi$ ) was then defined as follows:

$$
\mathcal{H}, h \models C \varphi \text { iff for each } h^{\prime} \in \mathrm{H} \text {, if } h \sim^{*} h^{\prime} \text { then } h^{\prime} \models \varphi
$$

Van Benthem, van Eijk and Kooi [33] discuss the technical issues that arise when axiomatizing Public Announcement Logic in languages with common knowledge. They introduce a new "relativized common knowledge" operator $C(\psi \mid \varphi)$ saying that all $\psi$-paths end in a states satisfying $\varphi$. More formally, with $\llbracket \varphi \rrbracket$ the set of histories satisfying $\varphi$, and $X^{+}$is the transitive closure of any set $X$ :

$$
\begin{gathered}
\mathcal{H}, h \models C(\psi \mid \varphi) \text { iff for each } h^{\prime} \in \mathrm{H}, \text { if }\left(h, h^{\prime}\right) \in\left(\bigcup_{i \in \mathcal{A}} \sim_{i} \cap(\mathrm{H} \times \llbracket \varphi \rrbracket)\right)^{+} \text {, then } \\
\mathcal{H}, h^{\prime} \models \psi
\end{gathered}
$$

The usual common knowledge operator $C \varphi$ can be defined as $C(T \mid \varphi)$. [33] axiomatizes public announcement logic with this additional operator, and shows how 
similar ideas work for DEL as a whole. We conjecture that the class $\mathbb{F}\left(\mathbf{X}_{P A L}\right)$ can be axiomatized by adding the following axiom to TPAL:

$$
\langle A\rangle C(\psi \mid \varphi) \leftrightarrow\langle A\rangle T \wedge C(\langle A\rangle \psi \mid\langle A\rangle \varphi)
$$

It is not hard to see that the above axiom is valid on the class $\mathbb{F}\left(\mathbf{X}_{P A L}\right)$.

There are other interesting notions of group knowledge. The most well-known is distributed knowledge expressing what a group knows if agents in the group share with the others everything they currently know. Formally, distributed knowledge of $\varphi$ (written $D \varphi)$ is defined using an intersection of accessibilities:

$$
\mathcal{H}, h \models D \varphi \text { iff for each } h^{\prime} \text {, if } h \sim_{i} h^{\prime} \text { for each } i \in \mathcal{A} \text {, then } \mathcal{H}, h^{\prime}=\varphi \text {. }
$$

As is well-known, there are a number of technical issues that arise when distributed knowledge is part of the language. For example, this notion is not bisimulation invariant, and complexity of validity tends to go up. We will need an extension of $\mathcal{L}_{E T L}$ with distributed knowledge in Section 6.1, as this notion naturally arises with communication protocols. For now, we conjecture that TPAL plus the usual rules for the modal operator $D$ and the following axiom scheme:

$$
\langle A\rangle \bar{D} \varphi \leftrightarrow(\langle A\rangle \top \wedge \bar{D}\langle A\rangle \varphi)
$$

is sound and complete for the class $\mathbb{F}\left(\mathbf{X}_{P A L}\right)$, where $\bar{D} \varphi$ is $\neg D \neg \varphi$.

Temporal Operators. Considering the "local" DEL and PAL logics from the "global" viewpoint of ETL makes it natural to extend the languages with temporal operators. We list below the definitions of a number of these. Let $\mathcal{H}=\left\langle\Sigma, H,\left\{\sim_{i}\right.\right.$ \}$\left._{i \in \mathcal{A}}, V\right\rangle$ is an ETL model, where $h \in \mathrm{H}$. If $e \in \Sigma$ and $n$ a natural number, then $e^{n}$ is the sequence of $e e \cdots e$ of length $n$. We define:

- $\mathcal{H}, h \models F \varphi$ iff there exists $h^{\prime} \in \mathrm{H}, h \preceq h^{\prime}$ and $\mathcal{H}, h^{\prime} \models \varphi$.

- $\mathcal{H}, h \models\left\langle e^{*}\right\rangle \varphi$ iff there is an $h^{\prime} \in \mathrm{H}$ with $h^{\prime}=h e^{n}$ for some $n$ and $\mathcal{H}, h^{\prime} \models \varphi$

- $\mathcal{H}, h \models N \varphi$ iff there is an $h^{\prime} \in \mathrm{H}$ with $h^{\prime}=h e$ for some $e \in \Sigma$ and $\mathcal{H}, h^{\prime}=\varphi$

- $\mathcal{H}, h \models\left\langle e^{-}\right\rangle \varphi$ iff there exists $h^{\prime} \in \mathrm{H}$ such that $h^{\prime} \prec_{e} h$ and $\mathcal{H}, h^{\prime}=\varphi$

- $\mathcal{H}, h \models P \varphi$ iff there is some $h^{\prime} \preceq h$ such that $\mathcal{H}, h^{\prime} \models \varphi$

We write $G \varphi$ for $\neg F \neg \varphi$ (i.e., $G \varphi$ means $\varphi$ is true in all future extensions). In fact, some work in this area has already been done, by studying extensions of the logics with the next-time operator $N$ or the Kleene star on events $\left\langle e^{*}\right\rangle$.

Let ProtocolPAL be the set of all finite sequences of public announcement event models (i.e., the full tree of all possible sequences of PAL event models). Similarly, 
let ProtocolDEL be the set of all finite sequences of event models. The usual axiomatization of public announcement logic works for the class $\mathbb{F}(\{$ ProtocolPAL $\})$ Similarly, the logic of $\mathbb{F}(\{$ ProtocolDEL $\})$ is the standard axiomatization of DEL [4, 33]). The following table summarizes what we know about complete logics for such languages with forward looking modalities (F.A. stands for 'Finite Axiomatizable' and EPDL stands for epistemic propositional dynamic logic. See [33] for details.):

\begin{tabular}{l|l|l}
\hline Language & $\mathbb{F}$ (ProtocolPAL) & $\mathbb{F}$ (ProtocolDEL) \\
\hline \hline$[i],\langle e\rangle$ & F.A. [23] & F.A. $[4]$ \\
\hline$[i],\langle e\rangle, C$ & F.A. [4] & F.A. [4] \\
\hline$E P D L,\langle e\rangle$ & F.A. [33] & F.A. [33] \\
\hline$[i],\langle e\rangle, N$ & F.A. [2] & Open \\
\hline$[i],\langle e\rangle,\left\langle e^{*}\right\rangle$ & Not F.A. [19] & Open \\
\hline$[i],\left\langle e^{*}\right\rangle$ & Open & Open \\
\hline$[i],\langle e\rangle, C,\left\langle e^{*}\right\rangle$ & Not F.A. [19] & Open \\
\hline
\end{tabular}

Miller \& Moss [19] show that $\mathbb{F}_{\mathcal{E}_{0}}^{\infty}=\left\{\operatorname{Forest}\left(M, \mathcal{E}_{0}\right) \mid M\right.$ infinite $\}$ where $\mathcal{E}_{0}=$ $\left\{\neg K_{i} \neg \top\right\}^{*}$ is not even axiomatizable for languages that contain knowledge modalities and arbitrary future modalities. There is some recent work axiomatizing the above classes with various backwards-looking modalities (see [35] and [24] for details). Even so, open problems abound here (cf. [32] and [13]).

The closest in spirit to the present paper is a recent paper by Balbiani et al. [2]. In [2], the operator $\diamond \varphi$ which is intended to mean "after any sequence of public announcements, $\varphi$ is true" is investigated. Formally, let $\mathcal{M}$ be an epistemic model and $w \in D(\mathcal{M})$, then we define:

$$
\mathcal{M}, w \models \diamond \varphi \text { iff there is a formula } \psi \in \mathcal{L}_{E L} \mathcal{M}, w \models\langle\psi\rangle \varphi .
$$

Among other interesting results, a complete axiomatization is provided for the class $\mathbb{F}(\{$ ProtocolPAL $\})$ with respect to the language containing epistemic modalities, public announcement modalities and the above "arbitrary announcement" modality [2]. These ideas merge naturally with ours. In particular, Hoshi [16] incorporates the idea of this operator into the current setting (i.e., by allowing the underlying protocol to vary) by considering an extension of $\mathcal{L}_{E T L}$ with the arbitrary future operator $(F)$.

\section{Logics of Specific Protocols}

Now that we have the general correspondence and axiomatization results of this paper, one next area of investigation should be the more detailed study of the behaviour of specific types of protocol, and the logical validities which they induce. 
What follows are just a few samples of such a logical protocol theory, to show its viability. Much of the discussion in this Section has been inspired by [10], who studies some other simple conversational-style restrictions. Also relevant is [28] on algorithms for 'maximal communication' eventually turning distributed knowledge into common knowledge. Similar procedures have been proposed by now for creating common beliefs out of individual beliefs, or (social-choice style)shared group preferences out of preferences of single agents. But the epistemic setting still seems a good logical pilot case. Also, though we will phrase our discussion in terms of communication protocols, the reader is invited to transpose our notions and results to observation-based or learning-based settings.

\subsection{Honest communication and the communicative core}

A minimal requirement for an "honest" public announcement, studied linguistic speech act theory, is that the speaker of the announcement believes what he announces. If we assume that all public announcements are made by an agent in the system, this means that an honest public announcement of $\varphi$ can be represented as a public announcement with a precondition $\varphi \wedge[i] \varphi$ for some $i \in \mathcal{A}$. More formally, an honest public announcement of $\varphi$ by agent $i$ is the event model $\mathcal{E}_{i, \varphi}=\left\langle\{e\},\left\{\longrightarrow_{i}\right\}_{i \in \mathcal{A}}\right.$, pre $\rangle$ with $e \longrightarrow_{i} e$ for all $i \in \mathcal{A}$ and $\operatorname{pre}(e)=\varphi \wedge[i] \varphi$. Thus, one interesting special protocol is that of honest communication, which uses all and only public announcements with preconditions of this form by ProtocolHonest.

"Runs" of this protocol can be expected to satisfy a kind of "safety property" - because all information being announced is already known by one of the agents, and hence the knowledge present in the whole system should neither increase or decrease if the protocol is followed. What such runs achieve in general, though, is increasing the shared group knowledge of such facts (modulo some complications having to do with epistemic non-factual assertions). To make this precise, we need to discuss first what we mean by "the knowledge present in a system." Recall the earlier definition of "distributed knowledge" $(D \varphi)$ :

- $\mathcal{M}, w \models D \varphi$ iff for each $w^{\prime}$ with $w \sim_{i} w^{\prime}$ for all $i \in \mathcal{A}$, then $\mathcal{M}, w^{\prime} \models \varphi$.

Distributed knowledge takes a snapshot of what agents, together, know or believe at a certain point in time. If each of them were to transfer all of their information to an outside agent, the information of this outsider corresponds with the distributed knowledge. ${ }^{10}$ The definition does not, however, take into account the fact that when agents communicate, the set of sentences that they believe to be true not

\footnotetext{
${ }^{10}$ This observation points at representations of group knowledge by introducing new agents, a device also suggested by McCarthy for common knowledge.
} 
only grows, but changes as well: first, I knew that I did not know $p$, but now I know that I do.

In [10] and [28] an alternative definition is proposed that does take this dynamic character into account. Let $\mathcal{M}=\left\langle W,\left\{R_{i}\right\}_{i \in \mathcal{A}}, V\right\rangle$ be an epistemic model with the $R_{i}$ all equivalence relations, and $w \in W$, then the "combined knowledge" or the "communicative core" at $w$ is the submodel $\left.\mathcal{M}\right|_{w} ^{I}$ of $\mathcal{M}$ whose states are only those worlds $w^{\prime}$ with $w R_{i} w^{\prime}$ for each agent $i$. Now we define a corresponding modal operator $I$ as follows:

- $\mathcal{M}, w \models I \varphi$ iff for each $w^{\prime} \in D\left(\left.\mathcal{M}\right|_{w} ^{I}\right)$ with $w R_{i} w^{\prime},\left.\mathcal{M}\right|_{w} ^{I}, w^{\prime} \models \varphi$

So, the set of states that make up the communicative core is the same as that for distributed knowledge - but we now view them in the context of the smaller model that has these states only. In [10] a sound and complete axiom system for the classical epistemic language with this new operator is defined.

The following proposition makes our previous intuition of 'information conservation' precise. It expresses that the communicative core in a system remains constant in the the protocol of honest announcements:

Proposition 6.1 For all $\mathcal{M}$ in which all $R_{i}$ are equivalence relations, and each $\varphi$ that is purely epistemic (that is, it has no temporal operators):

$$
\text { Forest }(\mathcal{M} \text {, ProtocolHonest }) \models I \varphi \leftrightarrow G I \varphi
$$

Proof. Let $\mathcal{M}=\left\langle W,\left\{R_{i}\right\}_{i \in \mathcal{A}}, V\right\rangle$ be an $S 5$ epistemic model ${ }^{11}$, and $\mathcal{E}$ a public announcement event model with precondition $\varphi \wedge[i] \varphi$. Suppose that $s$ is a state in $\mathcal{M}$ such that $s e \in \mathcal{M} \otimes \mathcal{E}$. We show that the communicative core in $\mathcal{M} \otimes \mathcal{E}$ is the same as that of $\mathcal{M}$. The desired result then follows by induction and the way that Forest $(\mathcal{M}$, ProtocolHonest) is constructed.

Let $\mathcal{M}^{\prime}=\mathcal{M} \otimes \mathcal{E}=\left\langle W^{\prime},\left\{R_{i}^{\prime}\right\}_{i \in \mathcal{A}}, V^{\prime}\right\rangle$. Our initial model $\mathcal{M}$ is an $S 5$ model, so $\mathcal{M}^{\prime}$ will be $\mathrm{S} 5$ as well. Let $s$ be any state in $\mathcal{M}$ such that $s e \in \mathcal{M}^{\prime}$. Because se $\in \mathcal{M}^{\prime}$, it must $\mathcal{M}, s \models \varphi \wedge[i] \varphi$. Since $\mathcal{M}$ is an $S 5$ model, it must be the case that $\varphi \wedge[i] \varphi$ is true in all states $s^{\prime}$ such that $s R_{i} s^{\prime}$. So, a fortiori, if $s R_{i} s^{\prime}$ for all $i$, then $\varphi \wedge[i] \varphi$ is true in $s^{\prime}$ as well. That means that there is an $s^{\prime} e \in \mathcal{M}^{\prime}$ such that $s e R_{i}^{\prime} s^{\prime} e$ for each $i$. By definition of product update, we also have that, if $s e \sim_{i} s^{\prime} e$ for each $i$, then $s \sim_{i} s^{\prime}$ for each $i$ in the original model. This means that the relation $Z$ given by $s Z$ se is an isomorphism between $\left.\mathcal{M}\right|_{s} ^{I}$ and $\left.\mathcal{M}^{\prime}\right|_{s e} ^{I}$, and therefore that $\mathcal{M}, s=I \varphi$ iff $\mathcal{M}^{\prime}$, se $=I \varphi$.

QED

\footnotetext{
${ }^{11}$ That is, an epistemic model where all the relations are equivalence relations.
} 
This leaves open the other intuitive aspect of the situation, viz. that agents can usually reach the communicative core by various forms of communication. Indeed, van Benthem [28] shows how, in finite models with two agents, the communicative core can be reached by the successive agents telling 'all they know', though 'bisimulation contractions' may be needed along the way to re-normalize the current model. On the other hand, [12] shows how this may break down with more than two agents. Intuitively, one would like to say, in a language with a temporal operator, that the following principle of our temporal protocol language should be valid, with ' $F$ ' a temporal future operator:

$$
D \varphi \rightarrow F C \varphi
$$

But the precise set of announcement protocols where this 'conversion from distributed into common knowledge' is true remains to be determined.

\subsection{Communication over an insecure channel}

While we have mainly concentrated on PAL protocols in this paper, more realistic interactive protocols typically involve partial observation on the part of agents, and insecure channels of communication. This needs the full apparatus of DEL rather than PAL, to which our approach can be lifted. Indeed, DEL protocols can formalize well-known phenomena (cf. [21, 28]) such as the classic "coordinated attack" problem ([8]), in which agents send each other messages over an insecure channel: messages are not guaranteed to arrive. We merely sketch how the style of analysis that we are advocating here lifts to this setting.

The epistemic effects of sending a message $\varphi$ that is not guaranteed to arrive can be represented by a DEL-style event model with three events, representing, respectively, the event $e_{1}$ where the message was sent and received, the event $e_{2}$ where the message was sent but not received, and the event $e_{3}$ in which no message was sent at all. Events $e_{1}$ and $e_{2}$ have as their precondition that the message $\varphi$ that was sent is believed to be true by the sender, i.e. $[s] \varphi$; while $e_{3}$ has just a trivial precondition $T$. The sender of the message cannot distinguish $e_{1}$ and $e_{2}$ (even if she knows that the message was sent, she does not know if the message arrived or not), the receiver cannot distinguish $e_{2}$ from $e_{3}$ (even if she knows no message arrived, she cannot know that this was because no message was sent in the first place, or because it got lost on the way).

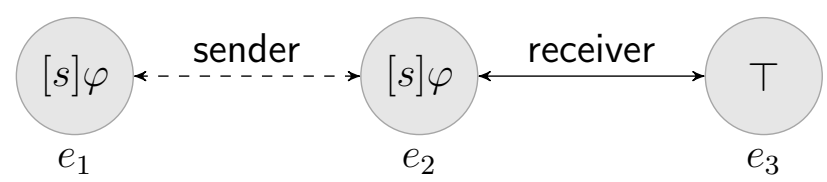


Now, let Protocollnsecure be the DEL protocol that contains all and only sequences of this type of message. We first discuss the situation informally, and then show how our logic captures the gist of it all. Since messages may actually arrive, agents can communicate, and learn new facts from each other by using this protocol. However, in S5 models, their common knowledge will never grow! For all propositional formulas, or, more generally, any formula $\varphi$ in which knowledge operators only occur under an even number of negations, either $\varphi$ is common knowledge throughout each run of the protocol, or it never is, was or will be.

However, even if common knowledge does not "grow", it may change. Even if no message is sent at all, it becomes common knowledge that the message $\varphi$ might have been sent. In particular, in a history where it is common knowledge that the receiver does not know that $p$, after an event of the form above, this fact will not be common knowledge any more. Even after $e_{3}$, in which no message was sent, it will still hold that, as far as the receiver knows, the sender might have sent a message, that, as far as the sender knows, the receiver might have received - and so, it will hold that $\neg K_{\text {receiver }} \neg \neg K_{\text {sender }} \neg[$ receiver $] p$.

Here is a more general logical fact behind all these observations:

Proposition 6.2 In all S5 models $\mathcal{M}$, the following holds for all formulas $\varphi$ in which epistemic operators occur only positively:

$$
\text { Forest }(\mathcal{M} \text {, Protocollnsecure }) \models C \varphi \leftrightarrow G C \varphi
$$

Proof. Let $C(\mathcal{M}, s)$ be the set of states $\left\{s^{\prime} \mid s R^{*} s^{\prime}\right\}$ (where $R^{*}$ is the reflexive transitive closure of the union of the $R_{i}$ ). We show that, if $\mathcal{E}$ is a communication over an insecure channel, and $e$ an event in $\mathcal{E}$, then $\mathcal{M} \otimes \mathcal{E}$, se $=C \varphi$ iff $\mathcal{M}, s \models C \varphi$ for each $\varphi$ in which epistemic operators occur only positively. The desired result then follows by construction of Forest $(\mathcal{M}$, Protocollnsecure) and a simple inductive argument.

We define a simulation that connects each $s \in C(\mathcal{M}, s)$ to some $t \in C(\mathcal{M} \otimes$ $\left.\mathcal{E}, s e_{k}\right)$, and vice versa. The result follows then from standard modal logic.

The first simulation $R$ is simply given by $s R e_{3}$. To see that this is a simulation, observe that, if $s \sim_{i} s^{\prime}$, then $s e_{3} \sim_{i} s^{\prime} e_{3}$. The more difficult part is showing that $s^{\prime} e_{3} \in C\left(\mathcal{M} \otimes \mathcal{E}, s e_{k}\right)$ for any $s^{\prime} \in C(\mathcal{M}, s)$. We show it for the three cases $k \in\{1,2,3\}$. For $k=3$, the result is immediate. For $k=2$, note that $s e_{2} \sim_{\text {receiver }} s_{3}$ (since $\mathcal{M}$ is reflexive), and since $C\left(\mathcal{M} \otimes \mathcal{E}, s e_{3}\right) \subseteq C\left(\mathcal{M} \otimes \mathcal{E}, s e_{2}\right)$, we are done by the previous case. Finally, consider $k=1$. Then (since the update succeeded) $\mathcal{M}, s \models[$ sender $] \varphi$, and hence $s e_{1} \sim_{\text {sender }} s e_{2}$. By the previous case, we have $s e_{2} \sim_{\text {receiver }} s e_{3}$, and so $s^{\prime} e_{3} \in C\left(\mathcal{M} \otimes \mathcal{E}, s e_{1}\right)$.

A simulation in the other direction is immediate, setting $s e_{k} R s$. For if $s e_{k} \sim_{i}$ $s^{\prime} e_{k^{\prime}}$, the definition of product update gives $s \sim_{i} s^{\prime} . \quad$ QED 
This outcome differs from a similar result by [8] which demonstrates that, under somewhat different assumptions, common knowledge will not change at all when communication is not reliable. Their stronger result depends on the assumption that the system is asynchronous - agents that do not receive a message also do not know that a message might have been sent.

These two case studies may have sufficed for showing the interest of a more general logic of protocols in a TPAL setting.

\section{Conclusions}

Epistemic-temporal logic and dynamic-epistemic logic are two important and interestingly different ways of describing knowledge-based agent interaction over time. We have shown how the two can be linked in three ways: using structural representation theorems, correspondence analysis for suitable modal languages, and new sorts of axiomatic completeness theorems for epistemic-temporal model classes generated by DEL protocols. Our results suggest a more systematic 'logic of protocols' using ideas from DEL to add fine-structure to ETL.

In our view, the two approaches complement each other: both in the way that models are constructed ("globally" in the ETL approach, "locally" from an initial model in the DEL approach), but also in the kind of models that are constructed. As we have seen, the ETL models that are generated by DEL protocols are a proper subclass of the full set - for example, asynchronous systems cannot be described by DEL updates. On the other hand, in the model constructions in [8] and [22], the assumption that epistemic relations are equivalence relations is more or less built in, while DEL also handles cases where information may be false.

Our framework also raises many new open problems. We mention issues of:

1. System Comparison: Can TPAL be embedded faithfully into PAL?

2. Complexity: Theorem 4.25 shows that the satisfiability problem for TPAL is decidable. What is its precise computational complexity?

3. Language Extensions: Section 5 discusses extensions of the language $\mathcal{L}_{T P A L}$ with common and distributed knowledge for groups. What about complete axiomatizations? (Section 5 suggests some axioms.) Also, our version of TPAL assumes that the statements that can be announced come only from the epistemic base language. What if we lift this restriction?

4. Partial Observation: The TPAL framework in this paper seems to generalize to DEL in general, with simple corresponding twists to axioms. What are the answers to the preceding questions then? Also, just when can protocol 
information be encoded completely in the DEL preconditions, as was the case for 'honest communication'?

5. Protocol Logic: Section 6.1 discusses logics of specific protocols, such as honest communication. What is its complete logic of the protocol class $\mathbb{F}$ (ProtocolHonest)? ${ }^{12}$ A number of recent papers has raised similar issues (see, for example, Baltag [?] and van Eijk [?]).

6. From Knowledge to Belief: Extend the analysis in this paper to doxastic logic and agents' changing beliefs over time. In particular, investigate protocols in doxastic logic, using doxastic-temporal logics and recent versions of DEL for belief change [26]. ${ }^{13}$

7. Learning Theory: Connect our logic of protocols with formal learning theory as in [17].

8. Process Theories: A final challenge that we see in the setting of this paper is using DEL, with its explicit account of model construction inside the logic, as an intermediate between ETL-style frameworks which describe properties of states and histories inside given models, and paradigms like process algebra or game semantics, with their explicit construction of dynamic processes.

In summary, we hope to have shown that the interface of DEL and ETL, as perhaps the two major current views of informative processes, is significant, productive, and well-worth exploring further.

\section{References}

[1] Abramsky, S., And Jagadeesan, R. Games and full completeness for multiplicative linear logic. Journal of Symbolic Logic 59, 2 (1994), 543 - 574.

[2] Balbiani, P., Baltag, A., van Ditmarsch, H., Herzig, A., Hoshi, T., AND DE LIMA, T. What can we achieve by arbitrary announcements? In Proceedings of TARK 2007 (2007), D. Samet, Ed.

[3] Baltag, A., And Moss, L. Logics for epistemic programs. Synthese: Knowledge, Rationality, and Action 2 (2004), 165 - 224.

\footnotetext{
${ }^{12}$ We conjecture that the logic of $\mathbb{F}$ (ProtocolHonest) (in the language $\mathcal{L}_{T P A L}$ ) is $T P A L$ with the axioms $\langle! \varphi \wedge[i] \varphi\rangle \top$ for all formulas $\varphi$, plus axioms of the form $\neg\langle\psi\rangle \top$ for all formulas $\psi$ that are not of the form $\varphi \wedge[i] \varphi$.

${ }^{13} \mathrm{An}$ analogue of our main representation theorem in terms of structural conditions and modal correspondence results has just been given in [29]
} 
[4] Baltag, A., Moss, L., And Solecki, S. The logic of public announcements, common knowledge and private suspicions. In Proceedings of TARK 1998 (1998).

[5] Belnap, N., Perloff, M., And Xu, M. Facing the Future. Oxford University Press, 2001.

[6] Blackburn, P., De Rijke, M., and Venema, Y. Modal Logic. Cambridge University Press, Cambridge, 2002.

[7] Bonanno, G. Memory and perfect recall in extensive games. Games and Economic Behaviour 47 (2004), 237 - 256.

[8] Fagin, R., Halpern, J., Moses, Y., And Vardi, M. Reasoning about Knowledge. The MIT Press, Boston, 1995.

[9] Gabbay, D., Kurucz, A., Wolter, F., And Zakharyaschev, M. Many-Dimensional Modal Logics: Theory and Applications. Elsevier, 2003.

[10] Gerbrandy, J. Bisimulations on Planet Kripke. PhD thesis, ILLC, 1999.

[11] Gerbrandy, J. Dynamic epistemic logic. In Logic, Language and Computation, L. S. Moss, J. Ginzburg, and M. de Rijke, Eds., vol. 2. CSLI Publications, Stanford, 1999, pp. 67-84.

[12] Gerbrandy, J. Convergence failures in public announcement scenarios. working paper, Department of Informatics, University of Torino, 2007.

[13] Halpern, J., van der Meyden, R., And Vardi, M. Complete axiomatizations for reasoning about knowledge and time. SIAM Journal of Computing 33, 2 (2004), $674-703$.

[14] Halpern, J., And VArdi, M. The complexity of reasoning about knowledge and time. J. Computer and System Sciences 38 (1989), 195 - 237.

[15] Hodkinson, I., And Reynolds, M. Temporal logic. In Handbook of Modal Logic, P. Blackburn, J. van Benthem, and F. Wolter, Eds. Elsevier, Amsterdam, 2006.

[16] Hoshi, T. Logics of public announcements with constrained protocols. Philosophy Department, Stanford University, 2007.

[17] Kelly, K. The Logic of Reliable Inquiry. Oxford University Press, 1996. 
[18] Lorini, E., And Castelfranchi, C. The cognitive structure of surprise: Looking for basic principles. Topoi 26 (2007), 133 - 149.

[19] Miller, J., And Moss, L. The undecidability of iterated modal relativization. Studia Logica 79, 3 (2005).

[20] Pacuit, E. Some comments on history based structures. J. Applied Logic 5, 4 (2007), 613-624.

[21] Pacuit, E., AND PARIKh, R. Reasoning about communication graphs. In Interactive Logic, Proceedings of the 7th Augustus de Morgan Workshop, J. van Benthem, D. Gabbay, and B. Löwe, Eds. King's College Press, 2007.

[22] Parikh, R., And Ramanujam, R. A knowledge based semantics of messages. Journal of Logic, Language and Information 12 (2003), 453 - 467.

[23] PlazA, J. Logics of public communications. In Proceedings, 4th International Symposium on Methodolgies for Intelligent Systems (1989).

[24] SACK, J. Temporal language for epistemic programs. Journal of Logic, Language and Information 17, 2 (2008), 183 - 216. Manuscript, Univeristy of Indiana, Bloomington.

[25] van Benthem, J. Games in dynamic epistemic logic. Bulletin of Economic Research 53 (2001), $216-248$.

[26] VAn Benthem, J. Dynamic logic for belief change. Journal of Applied Non-Classical Logics 14, 2 (2004).

[27] Van Benthem, J. Modal frame correspondences and fixed-points. Studia Logica 83 (2006), 133-155.

[28] van Benthem, J. One is a lonely number: on the logic of communication. In Logic Colloquium '02, Z. Chatzidakis, P. Koepke, and W. Pohlers, Eds. ASL \& A.K. Peters, 2006.

[29] VAn Benthem, J., And DÉGrémont, C. Building between dynamic and temporal doxastic logics. Tech. rep., ILLC, University of Amsterdam, 2008.

[30] van Benthem, J., And LiU, F. Diversity of logical agents in games. Philosophia Scientiae 8, 2 (2004), 163 - 178.

[31] van Benthem, J., And Martinez, M. The stories of logic and information. In Handbook of the Philosophy of Information, P. Adriaans and J. van Benthem, Eds. Elsevier, Amsterdam, 2008. 
[32] van Benthem, J., And Pacuit, E. The tree of knowledge in action: Towards a common perspective. In Proceedings of Advances in Modal Logic Volume 6, G. Governatori, I. Hodkinson, and Y. Venema, Eds. King's College Press, 2006.

[33] van Benthem, J., van EiJCK, J., And Kooi, B. Logics of communication and change. Information and Computation 204, 11 (2006), 1620 - 1662.

[34] van Ditmarsch, H., VAn Der Hoek, W., And Kooi, B. Dynamic Epistemic Logic. Synthese Library. Springer, 2007.

[35] Yap, A. Product update and looking backward. Manuscript, Stanford University, 2005. 